TRANSACTIONS OF THE

AMERICAN MATHEMATICAL SOCIETY

Volume 357, Number 9, Pages 3525-3551

S 0002-9947(04)03593-7

Article electronically published on November 4, 2004

\title{
CLONES FROM CREATURES
}

\author{
MARTIN GOLDSTERN AND SAHARON SHELAH
}

\begin{abstract}
We show that (consistently) there is a clone $\mathcal{C}$ on a countable set such that the interval of clones above $\mathcal{C}$ is linearly ordered and has no coatoms.
\end{abstract}

\section{INTRODUCTION}

A clone $\mathcal{C}$ on a set $X$ is a set of finitary operations $f: X^{n} \rightarrow X$ which contains all the projections and is closed under composition. (Alternatively, $\mathcal{C}$ is a clone if $\mathcal{C}$ is the set of term functions of some universal algebra over $X$.)

The family of all clones forms a complete algebraic lattice $C l(X)$ with greatest element $\mathcal{O}=\bigcup_{n=1}^{\infty} \mathcal{O}^{(n)}$, where $\mathcal{O}^{(n)}=X^{X^{n}}$ is the set of all $n$-ary operations on $X$. (In this paper, the underlying set $X$ will always be the set $\mathbb{N}=\{0,1,2, \ldots\}$ of natural numbers.)

The coatoms of this lattice $C l(X)$ are called "precomplete clones" or "maximal clones" on $X$.

For singleton sets $X$ the lattice $C l(X)$ is trivial; for $|X|=2$ the lattice $C l(X)$ is countable, and well understood ("Post's lattice"). For $|X| \geq 3, C l(X)$ has uncountably many elements. Many results for clones on finite sets can be found in 16. In particular, there is an explicit description of all (finitely many) precomplete clones on a given finite set ([10, see also 9] and [1]); this description also includes a decision procedure for the membership problem for each of these clones. It is also known that every clone $\mathcal{C} \neq \mathcal{O}$ is contained in a precomplete clone, that is, the clone lattice $C l(X)$ on any finite set $X$ is dually atomic. (This gives an explicit criterion for deciding whether a given set of functions generates all of $\mathcal{O}$; just check if it is contained in one of the precomplete clones.)

Fewer results are known about the lattice of clones on an infinite set: 3 investigated the interval of clones above the clone of unary functions on a countable set, [4] did this also for uncountable sets. [6] classified the countably many precomplete clones on a countable set that contain all bijections. [12 showed that there are always $2^{2^{\kappa}}$ precomplete clones on a set of infinite cardinality $\kappa$, and [11] gave specific examples of such precomplete clones. 7] investigated minimal clones.

Received by the editors March 7, 2003 and, in revised form, December 2, 2003.

2000 Mathematics Subject Classification. Primary 08A40; Secondary 03E50, 03E75.

Key words and phrases. Precomplete clones, maximal clones, clones on infinite sets, creature forcing, continuum hypothesis.

The first author is grateful to the Department of Mathematics, Rutgers University, New Jersey, for their hospitality during a visit in September 2002.

The second author's research was supported by the US-Israel Binational Science Foundation. Publication 808 .

A preprint of this paper is available at http://www.arXiv.org/math.RA/0212379/. 
[13] investigated "local" clones on infinite sets (clones that are closed sets in the product topology). It is easy to see that the lattice of local clones is far from being dually atomic ([5]).

Already Gavrilov in [2 pp. 22 and 23] asked whether the lattice of all clones on a countable set is also dually atomic, since a positive answer would be an important component for a completeness criterion, as remarked above. This question is also listed as problem P8 in [8, page 91], and has been open until now.

We will show here (assuming the continuum hypothesis, $\mathrm{CH}$ ) the following:

0.1. Theorem $(\mathrm{CH})$. The lattice of clones on a countably infinite set is not dually atomic, i.e., there is a clone $\mathcal{C} \neq \mathcal{O}$ which is not contained in any precomplete clone.

We also remark that the full strength of $\mathrm{CH}$ is not needed for this theorem.

The clone $\mathcal{C}_{U}$ that we construct has the additional feature that we can give a good description of the interval $\left[\mathcal{C}_{U}, \mathcal{O}\right]$. (See 5.7(2) and 5.8(d).) In particular, it will be a linear order without a penultimate element, in which every countable set has an upper bound.

All clones that we consider will be in the interval $\left[\mathcal{C}_{\text {id }}, \mathcal{O}\right]$, where $\mathcal{C}_{\text {id }}$ is the clone of all functions which are bounded by the max function. The clones in the interval $\left[\mathcal{C}_{\mathrm{id}}, \mathcal{O}\right]$ have the property that they are determined by their unary part. Moreover, the map that assigns to each clone its unary part is a lattice isomorphism between $\left[\mathcal{C}_{\mathrm{id}}, \mathcal{O}\right]$ and the set of all those monoids $\subseteq \mathbb{N}^{\mathbb{N}}$ which are lattice ideals (in the product order; see Definition 1.4).

Thus, our theorem can be reformulated as follows:

0.2. Theorem $(\mathrm{CH})$. The lattice of all submonoids of $\mathbb{N}^{\mathbb{N}}$ which are ideals is not dually atomic.

The method behind our proof is "forcing with normed creatures", a set-theoretic construction originating in the second author's paper [15. The book 14, an encyclopedia of such creatures, may be useful for constructing variants of our clone $\mathcal{C}_{U}$ to get clone intervals with prescribed properties; however, for the purposes of this paper the connection with forcing machinery is sufficiently shallow to allow us to be self-contained.

In particular, no knowledge of set theory is required for our theorem, except for Section 5, where a basic understanding of $\mathrm{CH}$ and transfinite induction up to $\omega_{1}$ is needed, and the discussion in Section [6] which is mainly directed at set theorists and not essential for the rest of the paper. Most of our constructions deal with finite structures, or with countable sequences of finite structures.

\section{Clones Defined By GROWth CONDitions}

\subsection{Notation.}

(1) $\mathcal{O}^{(1)}=\mathbb{N}^{\mathbb{N}}$ is the monoid of all functions from $\mathbb{N}$ to $\mathbb{N}$ (the operation is composition of functions). For $k \geq 1, \mathcal{O}^{(k)}$ is the set of all functions from $\mathbb{N}^{k}$ to $\mathbb{N}$, and $\mathcal{O}=\bigcup_{k=1}^{\infty} \mathcal{O}^{(k)}$.

(2) For $f, g \in \mathbb{N}^{\mathbb{N}^{k}}$, we write $f \leq g$ iff for all $k$-tuples $\vec{n}=\left(n_{1}, \ldots, n_{k}\right)$ we have $f(\vec{n}) \leq g(\vec{n})$.

(3) We write $f \leq^{*} g$ iff $f(\vec{n}) \leq g(\vec{n})$ holds for almost all (i.e., for all but finitely many) tuples $\vec{n}=\left(n_{1}, \ldots, n_{k}\right)$. In general, we use the superscript * or the keyword "almost" to indicate that finitely many exceptions are allowed. 
(4) id : $\mathbb{N} \rightarrow \mathbb{N}$ is the identity function.

(5) For $f \in \mathbb{N}^{\mathbb{N}}$, we write $f^{(n)}$ for the $n$-fold composition of $f$ with itself $\left(f^{(0)}=\right.$ id, $\left.f^{(1)}=f\right)$.

(6) $\max _{k}$ is the $k$-ary maximum function. Often we just write max.

(7) A growth function is a (not necessarily strictly) increasing function from $\mathbb{N}$ to $\mathbb{N}$ satisfying $f(n)>n$ for all $n$. We write $\mathcal{G}$ for the set of all growth functions.

(8) For any function $g: \mathbb{N}^{k} \rightarrow \mathbb{N}$ we let $\bar{g}: \mathbb{N} \rightarrow \mathbb{N}$ be defined by

$$
\bar{g}(n):=\max \left\{g\left(x_{1}, \ldots, x_{k}\right): x_{1}, \ldots, x_{k} \leq n\right\},
$$

and we let $\hat{g}(n)=\max \{n, \bar{g}(n)\}$. So $\hat{g}+1$ is a growth function.

(9) If $f$ is $k$-ary, $g_{1}, \ldots, g_{k}$ are $n$-ary, then we write $f\left(g_{1}, \ldots, g_{k}\right)$ for the function that maps $\vec{x}=\left(x_{1}, \ldots, x_{n}\right)$ to $f\left(g_{1}(\vec{x}), \ldots, g_{k}(\vec{x})\right)$. For example, $\max (g, h)$ is the pointwise maximum of $g$ and $h$.

(10) $\mathcal{C}_{\text {id }}:=\{g \in \mathcal{O}: g \leq \max \}=\{g \in \mathcal{O}: \bar{g} \leq \mathrm{id}\}$.

It is clear that $\mathcal{C}_{\mathrm{id}}$ is a clone. We will only consider clones that include $\mathcal{C}_{\mathrm{id}}$.

Note that the function $\bar{g}$ is always increasing (but not necessarily strictly). $\bar{g}$ measures the "growth" of $g$.

1.2. More notation. The following symbols are collected here only for easier reference:

- Relations between functions: $f \leq g, f \leq{ }^{*} g, f={ }^{*} g$; see 1.1

- Growth functions, $\mathcal{G}$; see 1.1

- The function $h_{A}$ and the relation $f \leq_{A} g$ for infinite sets $A$; see 1.13.

- The Ramsey function $\mathcal{R}$; see 2.4

- Relations between fronts or *fronts in zoos: $F \prec_{s} G, F \prec_{s}^{*} G, F \preccurlyeq_{s} G$, $F \preccurlyeq_{s}^{*} G, F \approx_{s}^{*} G$; see 4.9.

- More relations between fronts: $F+n \preccurlyeq_{s}^{*} G, F+n \approx_{s}^{*} G, F+\infty \preccurlyeq_{s}^{*} G$; again see 4.9

- Relations between growth functions $f$ and $g$ that are gauged by a zoo $s$ : $f \prec_{s} g, f \prec_{s}^{*} g, f \preccurlyeq_{s}^{*} g, f \approx_{s}^{*} g$; see 4.13

- The *fronts $F(s, f)$; see 4.5

- Relations between zoos: $t={ }^{*} s$ (see 3.3), $t \leq s, t \leq{ }^{*} s$ (see 3.9) $), t \leqq s$, $t \leqq * s ;$ see 3.10 .

- Tree order $\triangleleft, \triangleleft$; see 2.1 Lexicographic order $<$ on nodes in creatures (see 2.5), and on zoos; see 3.5 Direct lexicographic successor «; see 3.5

From clones to ideal monoids. We first show that above $\mathcal{C}_{\text {id }}$ we can restrict our attention to unary functions.

1.3. Lemma. Let $\mathcal{C}$ be a clone with $\mathcal{C}_{\mathrm{id}} \subseteq \mathcal{C}$. Then:

(1) $\mathcal{C}$ is downward closed: If $f \leq g$, and $g \in \mathcal{C}$, then $f \in \mathcal{C}$.

(2) For all $g: \mathbb{N}^{k} \rightarrow \mathbb{N}: g \in \mathcal{C}$ iff $\bar{g} \in \mathcal{C}$ iff $\hat{g} \in \mathcal{C}$. (See 1.1(8).)

(3) If the successor function id +1 (mapping each $x \in \mathbb{N}$ to $x+1$ ) is in $\mathcal{C}$, then we also have $g \in \mathcal{C}$ iff $\hat{g}+1 \in \mathcal{C}$.

Proof. (1) Let $g \in \mathcal{C}, g: \mathbb{N}^{k} \rightarrow \mathbb{N}$, and $f \leq g$. Define a $k+1$-ary function $F$ by

$$
F(\vec{x}, n)=\min (f(\vec{x}), n) \text {. }
$$

Clearly $F \in \mathcal{C}_{\text {id }}$, and $f(\vec{x})=F(\vec{x}, g(\vec{x}))$ for all $\vec{x}=\left(x_{1}, \ldots, x_{k}\right)$, so $f \in \mathcal{C}$. 
(2) Note that $g \leq \bar{g}\left(\max _{k}\right)$, and $\bar{g} \leq \hat{g}$, so $\hat{g} \in \mathcal{C} \Rightarrow \bar{g} \in \mathcal{C} \Rightarrow g \in \mathcal{C}$, as $\mathcal{C}$ is downward closed. The implication $\bar{g} \in \mathcal{C} \Rightarrow \hat{g} \in \mathcal{C}$ follows from $\max _{2} \in \mathcal{C}_{\text {id }}$.

It remains to check that $g \in \mathcal{C} \Rightarrow \bar{g} \in \mathcal{C}$ : So assume $g \in \mathcal{C}$. For each $n \in \mathbb{N}$ choose $\left(h_{1}(n), \ldots, h_{k}(n)\right) \leq(n, n, \ldots, n)$ such that

$$
g\left(h_{1}(n), \ldots, h_{k}(n)\right)=\max \left\{g\left(x_{1}, \ldots, x_{k}\right): x_{1}, \ldots, x_{k} \leq n\right\} .
$$

Then $h_{1}, \ldots, h_{k} \in \mathcal{C}_{\text {id }}$, so $\bar{g}=g\left(h_{1}, \ldots, h_{k}\right) \in \mathcal{C}$.

(3) The implication $\hat{g} \in \mathcal{C} \Rightarrow \hat{g}+1 \in \mathcal{C}$ follows because id $+1 \in \mathcal{C}$, the converse is true because $\mathcal{C}$ is downward closed.

1.4. Definition. A set $M \subseteq \mathcal{O}^{(1)}$ is an ideal monoid iff $M$ is both a monoid and a (lattice) ideal, i.e.,

(1) $(M, \circ$, id $)$ is a monoid,

(2) $M$ is downward closed: $\forall g \in M \forall f \leq g: f \in M$,

(3) $M$ is closed under max: $\forall f_{1}, f_{2} \in M: \max \left(f_{1}, f_{2}\right) \in M$. Using (1) and (2), this is equivalent to: $\forall f \in M: \max (f$, id $) \in M$.

Let $\mathcal{J}$ be the set of ideal monoids.

\subsection{Proposition.}

(1) $(\mathcal{J}, \subseteq)$ is a complete algebraic lattice, isomorphic to the interval $\left[\mathcal{C}_{\mathrm{id}}, \mathcal{O}\right]$ in the clone lattice.

(2) The map $\mathcal{C} \mapsto \mathcal{C} \cap \mathcal{O}^{(1)}$ is an isomorphism from $\left[\mathcal{C}_{\mathrm{id}}, \mathcal{O}\right]$ onto $\mathcal{J}$, with inverse $M \mapsto\{g \in \mathcal{O}: \bar{g} \in M\}$.

Proof. If $\mathcal{C} \supseteq \mathcal{C}_{\mathrm{id}}$ is a clone, then $\mathcal{C}$ is downward closed by 1.3(1) and contains the max function, so $\mathcal{C} \cap \mathcal{O}^{(1)}$ is an ideal monoid.

Conversely, if $M$ is an ideal monoid, then $\mathcal{C}(M):=\{g: \bar{g} \in M\}$ certainly contains $\mathcal{C}_{\text {id }}$. To check that $\mathcal{C}(M)$ is closed under composition of functions it is enough to verify:

$$
\text { If } h=g\left(f_{1}, \ldots, f_{k}\right) \text { and } \bar{f}_{1}, \ldots, \bar{f}_{k}, \bar{g} \in M \text {, then also } \bar{h} \in M,
$$

which follows from $\bar{h} \leq \bar{g}\left(\max \left(\bar{f}_{1}, \ldots, \bar{f}_{k}\right)\right)$.

The fact that the two maps are inverses of each other now follows easily from Lemma 1.3(2).

1.6. Remark. The isomorphism from $\mathcal{J}$ onto $\left[\mathcal{C}_{\mathrm{id}}, \mathcal{O}\right]$ can also be described by the map $M \mapsto \operatorname{Pol}(M)$, where (see [8]; we treat $M \subseteq X^{X}$ as an $X$-ary relation on $X$ )

$$
\operatorname{Pol}(M):=\bigcup_{k}\left\{g \in \mathcal{O}^{(k)}: \forall h_{1}, \ldots, h_{k} \in M: g\left(h_{1}, \ldots, h_{k}\right) \in M\right\} .
$$

We leave the verification to the reader.

So from now on we will only investigate ideal monoids instead of clones above $\mathcal{C}_{\text {id }}$. Our aim is to find an ideal monoid $M$ such that the interval $\left[M, \mathcal{O}^{(1)}\right]$ of ideal monoids (is linearly ordered and) has no coatom.

From ideal monoids to growth semigroups. The next step is mainly cosmetical; functions $f$ satisfying $f(n)=n$ for some $n$ are unpleasant to work with, so we want to ignore them.

Recall that $\mathcal{G}$ is the set of growth functions (see 1.117)). Note the following easy facts. 


\subsection{Fact.}

(a) If $f$ and $g$ are growth functions, then $f \circ g$ is a growth function.

(b) For any $n \in \mathbb{N}$, the set $M_{n}:=\left\{f \in \mathcal{O}^{(1)} \mid \forall k \leq n: f(k) \leq n\right\}$ is a coatom in the lattice of ideal monoids. (In clone theory, the set $M_{n}$ is usually called $\operatorname{Pol}(\{0, \ldots, n\})$.)

(c) The map $M \mapsto M \cap \mathcal{G}$, mapping an ideal monoid to its set of growth functions, is not $1-1$.

Proof. (a) is trivial, and (b) is easy and well known. $\emptyset$.

For (c), note that the sets $M_{n}$ from (b) are all different, but all satisfy $M_{n} \cap \mathcal{G}=$

These observations motivate us to restrict our attention to the set of those ideal monoids that contain the function $x \mapsto x+1$ (the smallest growth function).

1.8. Definition. A set $S \subseteq \mathcal{G}$ is called a growth semigroup iff $S$ is nonempty, closed under composition of functions, and (in $\mathcal{G}$ ) downward closed. (Since $\max (f, g) \leq$ $f \circ g$ holds for all growth functions $f$ and $g, S$ must also be closed under the max function.)

We write $\mathcal{C}_{\mathrm{id}+1}$ for the clone generated by $\mathcal{C}_{\mathrm{id}}$ together with the successor function $x \mapsto x+1$; thus $\mathcal{C}_{\mathrm{id}+1} \cap \mathcal{O}^{(1)}$ is the smallest ideal monoid containing the successor function, and $\mathcal{C}_{\mathrm{id}+1} \cap \mathcal{G}$ is the smallest growth semigroup.

1.9. Fact. The map $M \mapsto M \cap \mathcal{G}$ is an isomorphism from the interval $\left[\mathcal{C}_{\mathrm{id}+1} \cap\right.$ $\left.\mathcal{O}^{(1)}, \mathcal{O}^{(1)}\right]$ of ideal monoids onto the set of growth semigroups.

Proof. By Lemma $1.3(3)$.

So the interval $\left[\mathcal{C}_{\mathrm{id}+1}, \mathcal{O}\right]$ in the clone lattice is isomorphic to the lattice of growth semigroups. From now on we will only investigate growth semigroups. Our aim is to find a growth semigroup $S$ such that the interval $[S, \mathcal{G}]$ of growth semigroups (is linearly ordered and) has no coatom.

From growth semigroups to single growth functions. The next reduction is the most important one. Instead of investigating a lattice of growth semigroups (or clones, or ideal monoids), we can reduce our analysis to the investigation of the natural partial quasiorder " $g$ generates $f$ " of growth functions which (after factorization) turns out to be an upper semilattice. The interval of clones/monoids/semigroups that we are interested in will be naturally isomorphic to the set of ideals of this semilattice.

1.10. Fact. Let $S$ be a growth semigroup, $f$ and $g$ growth functions. Then the following are equivalent:

(1) $f$ is in the smallest growth semigroup containing $S \cup\{g\}$.

(2) There is a growth function $h \in S$ and a natural number $k$ such that $f \leq$ $(h \circ g)^{(k)}$.

(3) There is a growth function $h \in S$ and a natural number $k$ such that $f \leq$ $(\max (h, g))^{(k)}$.

(4) $f$ is in the clone generated by $S \cup \mathcal{C}_{\text {id }} \cup\{g\}$. 
Proof. The equivalence $(2) \Leftrightarrow(3)$ follows from $\max (h, g) \leq h \circ g \leq(\max (h, g))^{(2)}$. The implication " $(2) \Rightarrow(1)$ " follows from the closure properties of growth semigroups, and "(1) $\Rightarrow(2)$ " follows from the fact that, for any $g$, the set of growth functions $f$ satisfying (2) is a growth semigroup.

The equivalence of (4) to the other conditions is left to the reader.

1.11. Definition. If any/all of the above conditions (1), (2), (3), (4) are satisfied, we will write $f \leq_{S} g$, and we will write $\sim_{S}$ for the associated equivalence relation: $f \sim_{S} g$ iff $f \leq_{S} g$ and $g \leq_{S} f$. We write $\mathcal{G} / \sim_{S}$ or just $\mathcal{G} / S$ for the set of equivalence classes.

We have:

(a) $\leq_{S}$ is a partial quasiorder of functions.

(b) The set $S$ is the smallest $\sim_{S}$-equivalence class.

(c) The set $\mathcal{G} / S$ (with the order induced by $\leq_{S}$ ) is a join-semilattice.

Proof. It is clear that $\leq_{S}$ is transitive and reflexive. Clause (1) in the definition of $\leq_{S}$ easily implies that $S$ is the smallest equivalence class.

We will write $f / S$ for the $\sim_{S}$-class of $f$. To show clause (c) we prove that the class $\max \left(f_{1}, f_{2}\right) / S$ is the least upper bound of the classes $f_{1} / S$ and $f_{2} / S$. Clearly $\max \left(f_{1}, f_{2}\right) / S$ is an upper bound; if $g / S$ is also an upper bound, then we have $f_{1} \leq\left(\max \left(h_{1}, g\right)\right)^{\left(k_{1}\right)}$ and $f_{2} \leq\left(\max \left(h_{2}, g\right)\right)^{\left(k_{2}\right)}$ for some $h_{1}, h_{2} \in S, k_{1}, k_{2} \in \mathbb{N}$.

Now let $h:=\max \left(h_{1}, h_{2}\right) \in S$ and $k:=\max \left(k_{1}, k_{2}\right)$. So we have $\max \left(f_{1}, f_{2}\right) \leq$ $(\max (h, g))^{(k)}$, so $\max \left(f_{1}, f_{2}\right) \leq_{S} g$.

1.12. Fact. Fix a growth semigroup $S_{0}$.

Then for every growth semigroup $S \supseteq S_{0}$, the set $\left\{f / S_{0}: f \in S\right\}$ is an ideal in the semilattice $\mathcal{G} / S_{0}$.

Conversely, if $I \subseteq \mathcal{G} / S_{0}$ is a (nonempty) semilattice ideal, then $\left\{f \in \mathcal{G}: f / S_{0} \in\right.$ $I\}$ is a growth semigroup containing $S_{0}$.

Moreover, the maps defined in the previous two paragraphs are inverses of each other. Thus, the interval $\left[S_{0}, \mathcal{G}\right]$ in the set of growth semigroups is naturally isomorphic to the set of ideals on $\mathcal{G} / S_{0}$.

In particular, we get: If $\mathcal{G} / S_{0}$ is linearly ordered, then:

(1) The interval $\left[S_{0}, \mathcal{G}\right]$ in the lattice of growth semigroups corresponds exactly to the nonempty downward closed subsets of $\mathcal{G} / S_{0}$ (the "Dedekind cuts").

(2) The interval $\left[S_{0}, \mathcal{G}\right]$ has a coatom iff $\mathcal{G} / S_{0}$ has a greatest element.

Proof. Again this boils down to $\max (h, g) \leq h \circ g \leq(\max (h, g))^{(2)}$. The fact that semigroups are always unions of $\sim_{S_{0}}$-equivalence classes is clear if we use 1.10 1 ) to define $\leq_{S}$.

Conclusion and goals. We will construct a growth semigroup $S_{0}$ such that the partial order $\mathcal{G} / S_{0}$ will be a linear order with a smallest element where each element has a direct successor and (except for the smallest one) a direct predecessor; moreover, all countable sets will be bounded, and all intervals in this order will be either finite or uncountable.

The set of growth semigroups (or equivalently, the interval of clones) above $S_{0}$ will be the Dedekind completion of this order; it will be a linear order with no coatom. 
Replacing growth semigroups by filters. It turns out to be convenient to concentrate on growth functions of a certain kind, the functions $h_{A}$ defined below.

\subsection{Definition.}

(1) For any infinite set $A \subseteq \mathbb{N}$, we let $h_{A}$ be defined by

$$
h_{A}(n):=\min \{a \in A: a>n\} .
$$

We let $S_{A}$ be the growth semigroup generated by $h_{A}$.

(2) For any infinite set $A \subseteq \mathbb{N}$, and for any growth functions $f, g$ we write $f \leq_{A} g$ for $f \leq_{S_{A}} g$, or more explicitly:

$$
f \leq_{A} g: \Leftrightarrow \exists k: f \leq\left(\max \left(g, h_{A}\right)\right)^{(k)}
$$

(equivalently: there is a $k$ such that $\left.f \leq\left(g \circ h_{A}\right)^{(k)}\right)$.

(3) If $U$ is a filter of infinite subsets of $\mathbb{N}$, we let $f \leq_{U} g$ iff there is $A \in U$ with $f \leq_{A} g$.

(4) If $U$ is as above, we let $\mathcal{G}_{U}:=\left\{g \in \mathcal{G}: g \leq_{U}\right.$ id $\}=\{g \in \mathcal{G}: \exists A \in U \exists k$ : $\left.g \leq h_{A}^{(k)}\right\}$. (Note that id $+1=h_{\mathbb{N}} \in \mathcal{G}_{U}$ for any $U$.)

\subsection{Fact.}

(1) $h_{A}$ is a growth function. Conversely, for any (growth) function $g: \mathbb{N} \rightarrow \mathbb{N}$ there is a set $A$ such that $g \leq h_{A}^{(2)}$.

(2) If $A \subseteq B$, then $h_{B} \leq h_{A}$, so $f \leq_{B} g$ implies $f \leq_{A} g$. Hence $h_{A \cap B} \geq$ $\max \left(h_{A}, h_{B}\right)$ (if $A \cap B$ is infinite).

(3) If $A={ }^{*} B$, then the relations $\leq_{A}$ and $\leq_{B}$ coincide.

Therefore, if $U$ is a filter of infinite sets, then $\mathcal{G}_{U}$ is the smallest growth semigroup containing $\left\{h_{A}: A \in U\right\}$. Moreover, the relations

$$
f \leq_{\mathcal{G}_{U}} g
$$

(defined in 1.11), and

$$
f \leq_{U} g
$$

(defined in $1.13(3)$ ) are equivalent.

Outline of the proof of Theorem 0.1 . The relations $\leq_{A}$ and $\leq_{U}$ are partial quasiorders (i.e., reflexive and transitive relations) on $\mathcal{G}$.

Factoring out by the relation

$$
f \sim_{U} g \Leftrightarrow f \leq_{U} g \& g \leq_{U} f
$$

will thus give a partial order $L_{U}$.

We will find a filter $U$ (in fact, $U$ will be an ultrafilter) such that the relation $\leq_{U}$ is a linear quasiorder on $\mathcal{G}$, so $L_{U}:=\mathcal{G} / \sim_{U}$ will be linearly ordered. The smallest element in this order is the equivalence class of the function id, i.e., the set $\mathcal{G}_{U}$.

(It is not necessary to have this interval linearly ordered in order to obtain a clone $\mathcal{C}$ with $[\mathcal{C}, \mathcal{O}]$ not dually atomic, but it will make the proof more transparent.)

How do we construct $U$ ? For each pair of growth functions $f, g$ we want to have a set $A \in U$ witnessing $f \leq_{A} g$ or $g \leq_{A} f$. This requirement tends to add very "thin" sets to $U$ (since we have to add a set $A$ such that some iterate of $\max \left(g, h_{A}\right)$ dominates $f$ ).

Achieving this goal alone (under $\mathrm{CH})$ would be a trivial exercise: Let $\left(f_{i}, g_{i}\right.$ : $i \in \omega_{1}$ ) be an enumeration of all pairs of growth functions, then we can define a 
$\subseteq^{*}$-decreasing sequence $\left(A_{i}: i \in \omega_{1}\right)$ of infinite subsets of $\mathbb{N}$, making $A_{i}$ so thin that $f_{i} \leq A_{i} g_{i}$ or the converse holds. We then let $U$ be the filter generated by the sets $A_{i}$.

However, such a naive construction will make the sets $A_{i}$ so thin that the family $\left\{h_{A}: A \in U\right\}$ might dominate all unary functions, which would result in $\mathcal{G}_{U}=\mathcal{G}$.

So we will modify this naive construction. Together with the sets $A_{i}$ we will construct objects $s_{i}$ which place a limit on how fast the sets $A_{i}$ may be thinned out. These auxiliary objects $s_{i}$ will help to give a more explicit description of the relation $\leq_{A_{i}}$ (see 4.15 and 4.16).

\section{Trees And CREatures}

2.1. Definition. For any finite partial order $(T, \unlhd)$ we let $\operatorname{ext}(T)$ be the set of maximal elements of $T$, and $\operatorname{int}(T):=T \backslash \operatorname{ext}(T)$.

We let $x \triangleleft y$ iff $x \unlhd y$ and $x \neq y$.

For $\eta \in T$ we let $\operatorname{succ}_{T}(\eta)$ be the set of direct successors of $\eta$ in $T$, i.e.,

$$
\operatorname{succ}_{T}(\eta)=\left\{\nu \in T: \eta \triangleleft \nu, \neg \exists \nu^{\prime}\left(\eta \triangleleft \nu^{\prime} \triangleleft \nu\right)\right\}
$$

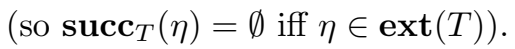

We say that $(T, \unlhd)$ is a tree iff

- $T$ is a finite set,

- $\unlhd$ is a partial order on $T$, with a least element, called $\operatorname{root}(T)$, and

- for all $\nu \in T$ the set $\{\eta: \eta \unlhd \nu\}$ is linearly ordered by $\unlhd$.

Elements of a tree $T$ will be called "nodes", elements of $\operatorname{int}(T)$ are "internal nodes".

Elements of $\operatorname{ext}(T)$ are called "leaves" or "external nodes".

For any $\eta \in T$ we let

$$
T^{[\eta]}:=\{\nu: \eta \unlhd \nu\}
$$

(with the induced order). If $T$ is a tree, then also $T^{[\eta]}$ is a tree, with root $\eta$.

2.2. Definition. Let $(T, \unlhd)$ be a tree. A branch of $T$ is a maximal linearly ordered subset of $(T, \unlhd)$. In other words, a branch is a set of the form $\{\eta: \eta \unlhd \nu\}$ for some $\nu \in \operatorname{ext}(T)$. (We may occasionally identify a node $\nu \in \operatorname{ext}(T)$ with the branch $\{\eta: \eta \unlhd \nu\}$.)

A front is a subset of $T$ which meets each branch exactly once. For example, $\operatorname{ext}(T)$ is a front, and the singleton $\{\operatorname{root}(T)\}$ is also a front. If $F \subseteq \operatorname{int}(T)$ is a front, then also $\bigcup_{\eta \in F} \operatorname{succ}_{T}(\eta)$ is a front.

Note that for any tree $(S, \unlhd$ ), any subset $T \subseteq S$ containing $\operatorname{root}(S)$ (with the induced order) will again be a tree. The following definition singles out some of those subsets.

2.3. Definition. If $(S, \unlhd)$ is a tree, $T \subseteq S$, we call $T$ a subtree of $S$ (" $T \leq S$ ") iff $T$ contains the root of $S$ and $\forall \eta \in T \cap \operatorname{int}(S): \emptyset \neq \operatorname{succ}_{T}(\eta) \subseteq \operatorname{succ}_{S}(\eta)$.

$(T, \unlhd)$ will again be a tree, and we have $\operatorname{ext}(T) \subseteq \operatorname{ext}(S), \operatorname{int}(T) \subseteq \operatorname{int}(S)$.

Below we will need the following version of Ramsey's theorem, a well-known theorem from finite combinatorics. 


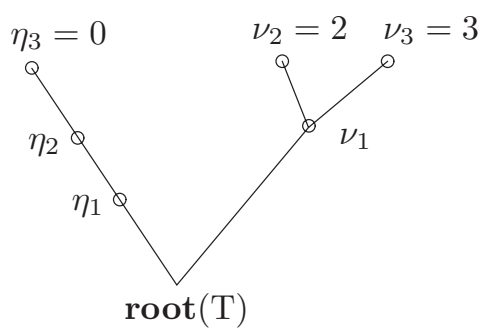

FiguRe 1.

2.4. Fact. For every natural number $n$ there is a natural number $k=\mathcal{R}(n)$ such that:

Whenever $f:[C]^{2} \rightarrow\{0,1\}$, with $|C| \geq k$,

there is a subset $A \subseteq C$ with $|A| \geq n$ which is homogeneous for $f$, i.e., such that $f \uparrow[A]^{2}$ is constant.

Here, $[C]^{2}:=\{\{x, y\}: x, y \in C, x \neq y\}$ is the set of all unordered pairs from $C$.

It is well known that one can choose $\mathcal{R}(n)=4^{n}$ or even smaller, but we do not need any good bounds on $\mathcal{R}(n)$, its mere existence is sufficient. For the rest of the paper we fix such a function $\mathcal{R}$.

Note that $\mathcal{R}$ will satisfy $\mathcal{R}(n) \geq 2 n$ for all $n \geq 3$. For technical reasons we will use a function $\mathcal{R}$ that satisfies $\mathcal{R}(n) \geq 2 n$ also for $n=1,2$. Thus we also have for all $n \geq 1$ :

Whenever $f: C \rightarrow\{0,1\}$, with $|C| \geq \mathcal{R}(n)$,

there is a subset $A \subseteq C$ with $|A| \geq n$ which is homogeneous for $f$, i.e., such that $f\lceil A$ is constant.

We let $\mathcal{R}^{-1}(k)=\max \{n: \mathcal{R}(n) \leq k\}$.

2.5. Definition. A creature is a finite tree $T$ where

- $\operatorname{ext}(T) \subseteq \mathbb{N}, \operatorname{int}(T) \cap \mathbb{N}=\emptyset$, and

- whenever $\eta_{1}, \eta_{2}$ are $\unlhd$-incomparable, then: either $\max \operatorname{ext} T^{\left[\eta_{1}\right]}<\min \operatorname{ext} T^{\left[\eta_{2}\right]}$, or else $\max \operatorname{ext} T^{\left[\eta_{2}\right]}<\min \operatorname{ext} T^{\left[\eta_{1}\right]}$.

$T$ is called improper if $T$ consists of the single external node $\operatorname{root}(T)$. Otherwise (i.e., if $\operatorname{root}(T) \in \operatorname{int}(T)$ ), $T$ is called a proper creature.

We will write $\max [\eta]$ and $\min [\eta]$ for $\max \operatorname{ext} T^{[\eta]}$ and $\min \operatorname{ext} T^{[\eta]}$, if the underlying tree $T$ is clear from the context. In particular, if $\eta \in \operatorname{ext}(T)$ then $T^{[\eta]}=\{\eta\}$, so $\max [\eta]=\min [\eta]=\eta$.

If $\eta_{1}, \eta_{2}$ are $\unlhd$-incomparable, we will write $\eta_{1}<_{T} \eta_{2}$ or just $\eta_{1}<\eta_{2}$ to abbreviate $\max \left[\eta_{1}\right]<\min \left[\eta_{2}\right] . \eta_{1} \leq \eta_{2}$ iff $\eta_{1}<\eta_{2}$ or $\eta_{1}=\eta_{2}$. This is a partial order.

We visualize this order as being embedded in the real plane $\mathbb{R}^{2}$, with the order $\unlhd$ pointing from the bottom to the top, whereas the order $\leq$ can be viewed as pointing from left to right. (See Figure 1, where we have $\eta_{1} \unlhd \eta_{2} \unlhd \eta_{3}, \nu_{1} \unlhd \nu_{2}$, $\nu_{1} \unlhd \nu_{3}, \nu_{2}<\nu_{3}$, and $\eta_{i}<\nu_{j}$ for all $i, j \in\{1,2,3\}$.)

We call $\leq$ the lexicographic order of nodes, since clearly

whenever $\eta_{1}<\eta_{2}$, and $\eta_{1} \unlhd \nu_{1}, \eta_{2} \unlhd \nu_{2}$,

then also $\nu_{1}<\nu_{2}$.

Note that on $\operatorname{ext}(T)$ this lexicographic order and the usual order of $\mathbb{N}$ agree. 
If $T$ is a proper creature, then we let $\|T\|:=\min \left\{\left|\operatorname{succ}_{T}(\nu)\right|: \nu \in \operatorname{int}(T)\right\}$. (If $T$ is improper, $\|T\|$ is undefined.)

2.6. Remark. (Readers not familiar with creature forcing are advised to skip this remark.) The reader who is familiar with 14 will be disappointed to see that norms, an essential ingredient in [14's creatures, are not mentioned here at all. It will become clear that for our purposes there is in fact a natural norm, namely $\operatorname{nor}_{T}(\eta):=\max \left\{k: \mathcal{R}^{(k)}(4) \leq\left|\operatorname{succ}_{T}(\eta)\right|\right\}$. See also 3.2 .

2.7. Notation. Let $E$ be a finite nonempty set of "colors" (often with only 2 elements).

We will consider three kinds of "coloring functions" on creatures $S$.

(1) (Partial) functions $c: S \rightarrow E$. These are called unary node colorings.

(2) (Partial) functions $c: \bigcup_{\eta \in \operatorname{int}(S)}\left[\operatorname{succ}_{S}(\eta)\right]^{2} \rightarrow E$. These are called $b i$ nary node colorings. We may write such functions more sloppily as $c$ : $[S]^{2} \rightarrow E$, with the understanding that we will only be interested in the values $c\left(\left\{\nu_{1}, \nu_{2}\right\}\right)$, where $\nu_{1}$ and $\nu_{2}$ have a common direct $\triangleleft$-predecessor.

(3) (Partial) functions $c: \operatorname{ext}(S) \rightarrow E$, or equivalently, functions $c$ whose domain is contained in the set of branches of $S$. These are called unary branch colorings.

If $c$ is a coloring of one of these types, and $T \leq S$, we say that $T$ is $c$-homogeneous (or homogeneous for $c$ ), iff:

(1) In the first case: $c\left\lceil\operatorname{succ}_{T}(\eta)\right.$ is constant, for all $\eta \in \operatorname{int}(T)$ (i.e., for all $\eta$ there is a color $e_{\eta}$ such that for all $\nu \in \operatorname{succ}_{T}(\eta)$ : either $c(\nu)$ is undefined, or $c(\nu)=e_{\eta}$.)

(2) In the second case: $c \uparrow\left[\operatorname{succ}_{T}(\eta)\right]^{2}$ is constant, for all $\eta \in \operatorname{int}(T)$.

(3) In the third case: $c \nmid \operatorname{ext}(T)$ is constant.

(The reader may wonder at this point why our colorings are only partial functions. Later we introduce an operation that produces new trees from old trees by gluing several old trees together, adding a new root. If the old trees are colored, this induces naturally a partial coloring function on the new tree, but the new root will not get a color.)

2.8. Lemma. Let $n \geq 3$, and let $S$ be a creature with $\|S\| \geq \mathcal{R}(n)$, let $E:=\{0,1\}$, and let $c: S \rightarrow E$, or $c:[S]^{2} \rightarrow E$, or $c: \operatorname{ext}(S) \rightarrow E$ be a coloring (with 2 colors) of one of the types mentioned above.

Then there is a creature $T \leq S,\|T\| \geq n$, which is homogeneous for $c$.

Similarly, if $c$ is a coloring with at most $2^{k}$ colors, and $\|S\| \geq \mathcal{R}^{(k)}(n)$, then we can find a c-homogeneous creature $T \leq S$ with $\|T\| \geq n$.

Proof. We will prove this only for the case of 2 colors. For $2^{k}$ colors, apply the argument for 2 colors $k$ times.

First and second case (unary and binary node colorings): We will define $T$ by ("upward") induction, starting with $\operatorname{root}(T):=\operatorname{root}(S)$. For any $\eta \in T$ we let $\operatorname{succ}_{T}(\eta)$ be a $c$-homogeneous subset of $\operatorname{succ}_{S}(\eta)$ of size $\geq n$. (Such a large homogeneous set exists $\operatorname{since} \operatorname{succ}_{S}(\eta)$ has at least $\mathcal{R}(n)$ elements.) This defines a creature $T$ with $\|T\| \geq n$, and it is clear that $T$ is $c$-homogeneous.

Third case: Let $c: \operatorname{ext}(S) \rightarrow\{0,1\}$. We will define a unary node coloring $c^{\prime}$ as follows, by ("downward") induction starting at the leaves:

- If $\eta \in \operatorname{ext}(S)$, then $c^{\prime}(\eta)=c(\eta)$. 
- If $\eta \in \operatorname{int}(S)$, and $c^{\prime}\left\lceil\operatorname{succ}_{S}(\eta)\right.$ is already defined, then we choose $c^{\prime}(\eta) \in$ $\{0,1\}$ such that

$$
\left|\left\{\nu \in \operatorname{succ}(\eta): c^{\prime}(\nu)=c^{\prime}(\eta)\right\}\right| \geq n .
$$

This is possible as $|\operatorname{succ}(\eta)| \geq \mathcal{R}(n) \geq 2 n$.

Now let $\ell_{0}:=c^{\prime}(\operatorname{root}(S))$, and define $T \leq S$ by requiring $\operatorname{root}(T)=\operatorname{root}(S)$, and

$$
\forall \eta \in T: \operatorname{succ}_{T}(\eta)=\left\{\nu \in \operatorname{succ}_{S}(\eta): c^{\prime}(\nu)=\ell_{0}\right\} .
$$

Then $\|T\| \geq n$, and $c^{\prime}$ is constant on $T$ with constant value $\ell_{0}$, so also $c$ is constant on $\operatorname{ext}(T)$.

\section{Zoos}

3.1. Definition. A zoo is a sequence $s=\left(S_{0}, S_{1}, \ldots\right)$ of proper creatures (see 2.5) such that:

- All $S_{n}$ are pairwise disjoint.

- For all $n, \max \operatorname{ext}\left(S_{n}\right)<\min \operatorname{ext}\left(S_{n+1}\right)\left(\right.$ recall that $\left.\operatorname{ext}\left(S_{n}\right) \subseteq \mathbb{N}\right)$.

- The sequence $\left(\left\|S_{n}\right\|: n \in \mathbb{N}\right)$ diverges to $\infty$, and $\forall n:\left\|S_{n}\right\| \geq 4$.

We define $\operatorname{ext}(s):=\bigcup_{n \in \mathbb{N}} \operatorname{ext}\left(S_{n}\right)$, similarly $\operatorname{int}(s)$.

We similarly transfer other notation from creatures to zoos, e.g., for $\eta \in S_{n}$ we may write $\operatorname{succ}_{s}(\eta)$ for $\operatorname{succ}_{S_{n}}(\eta), s^{[\eta]}$ for $S_{n}^{[\eta]}$, etc. For $\eta \in S_{n}$, we may write $\max [\eta]$ or $\max _{s}[\eta]$ for $\max \operatorname{ext}\left(S_{n}^{[\eta]}\right)$. We sometimes identify $s$ with $\operatorname{int}(s) \cup \operatorname{ext}(s)$, i.e., for $s=\left(S_{0}, S_{1}, \ldots\right)$ we write $\eta \in s$ instead of $\eta \in \bigcup_{n} S_{n}$.

3.2. Remark. (Again this remark is only for the benefit of readers familiar with [14.) With the definition $\operatorname{nor}(A):=\max \left\{k: \mathcal{R}^{(k)}(4) \leq|A|\right\}$ (for any finite set $A$ ), it is clear that $\lim _{n \rightarrow \infty} \operatorname{nor}\left(A_{n}\right)=\infty$ is equivalent to just $\lim _{n \rightarrow \infty}\left|A_{n}\right|=\infty$. This equivalence allows us to omit the definition of norms for our creatures.

3.3. Definition. Let $s=\left(S_{0}, \ldots\right)$ and $t=\left(T_{0}, \ldots\right)$ be zoos. We say $s={ }^{*} t$ if there are $n_{0}$ and $k_{0}$ such that $\left(S_{n_{0}}, S_{n_{0}+1}, \ldots\right)=\left(T_{k_{0}}, T_{k_{0}+1}, \ldots\right)$.

3.4. Definition. Let $t=\left(T_{0}, T_{1}, \ldots\right)$ be a zoo.

A branch of $t$ is a set which is a branch in one of the trees $T_{n}$.

A front is a set $F \subseteq t$ (i.e., $F \subseteq \bigcup_{n} T_{n}$ ) such that each of the sets $F \cap T_{n}$ is a front in $T_{n}$. Equivalently, $F$ meets every branch of $t$ in exactly one node.

A *front (or "almost front") is a set $F \subseteq \bigcup_{n} T_{n}$ such that for some $t^{\prime}={ }^{*} t, F \cap t^{\prime}$ is a front in $t^{\prime}$. Equivalently, $F$ is a ${ }^{*}$ front iff almost all (=all except finitely many) branches of $t$ meet $F$ in exactly one node.

If $F$ and $G$ are ${ }^{*}$ fronts in $t$, we write $F \approx^{*} G$ iff there is some $t^{\prime}={ }^{*} t$ such that $F \cap t^{\prime}=G \cap t^{\prime}$. (Equivalently, $F \approx^{*} G$ iff the symmetric difference of $F$ and $G$ is finite.)

3.5. Definition. Let $s=\left(S_{0}, S_{1}, \ldots\right)$ be a zoo. For $\eta, \nu \in \bigcup_{n} S_{n}$ we define

$$
\eta<\nu \Leftrightarrow \max [\eta]<\min [\nu]
$$

and we let $\eta \lessdot \nu$ iff $\eta$ and $\nu$ are on adjacent branches, i.e.,

$\eta<\nu$ and for all $k \in \operatorname{ext}(s): k \leq \max [\eta]$ or $k \geq \min [\nu]$.

For example, in Figure 1 we have $\eta_{i} \lessdot \nu_{j}$ for $i \in\{1,2\}$ and $j \in\{1,2\}$, but not for $j=3$.

The following fact is not needed, but can be useful to visualize fronts. 
3.6. Fact. Let $s=\left(S_{0}, S_{1}, \ldots\right)$ be a zoo. A set $F \subseteq \bigcup_{n} S_{n}$ is a front in $s$ iff $F$ can be enumerated as $F=\left\{\eta_{0}, \eta_{1}, \ldots\right\}$ where $\min \left[\eta_{0}\right]=\min \operatorname{ext} S_{0}$, and for all $n$ we have $\eta_{n} \lessdot \eta_{n+1}$.

Below we will several times have to generate new ("improved") zoos from old ones. The following examples are special cases of the general definition below.

3.7. Construction. Let $s=\left(S_{0}, S_{1}, \ldots\right)$ be a zoo.

drop: Let $n_{0}<n_{1}<\cdots$ be an infinite increasing sequence. Let $t=$ $\left(T_{0}, T_{1}, \ldots\right)$ be defined by $T_{k}=S_{n_{k}}$. Then $t$ is a zoo.

We say that $t$ is obtained from $s$ by dropping creatures (namely, by dropping the creatures $S_{i}$ with $\left.i \in \mathbb{N} \backslash\left\{n_{0}, n_{1}, \ldots\right\}\right)$.

shrink: For each $n$ let $T_{n} \leq S_{n}$ (see [2.3). Then $t=\left(T_{0}, T_{1}, \ldots\right)$ is a zoo, provided that $\lim _{n \rightarrow \infty}\left\|T_{n}\right\|=\infty$ and $\forall n:\left\|T_{n}\right\| \geq 4$.

We say that $t$ is obtained by shrinking $s$.

glue: Partition $\mathbb{N}$ into intervals: $\mathbb{N}=\bigcup_{k=0}^{\infty}\left[n_{k}, n_{k+1}\right)$ with $0=n_{0}<n_{1}<$ $n_{2}<\cdots$ and

$$
\lim _{k \rightarrow \infty}\left(n_{k+1}-n_{k}\right)=\infty, \quad \forall n: n_{k+1}-n_{k}>4 .
$$

For each $k$, let $T_{k}:=\left\{\tau_{k}\right\} \cup \bigcup_{i=n_{k}}^{n_{k+1}-1} S_{i}$, where $\tau_{k}=\operatorname{root}\left(T_{k}\right)$ will be a new element. Make $T_{k}$ into a creature by requiring: For all $i \in\left[n_{k}, n_{k+1}\right)$, all $\eta \in S_{i}: S_{i}^{[\eta]}=T_{k}^{[\eta]}$. Again $t$ will be a zoo.

Sometimes we have to compose the steps described above, but at one point (see 3.14) we will need a more complicated gluing process, as described in the following definitions.

3.8. Definition. A gluing recipe is a sequence $r=\left(R_{0}, R_{1}, \ldots\right)$ of (not necessarily proper) creatures satisfying $\forall k: \max \operatorname{ext}\left(R_{k}\right)<\min \operatorname{ext}\left(R_{k+1}\right)$, and moreover, if the set $A:=\left\{k: R_{k}\right.$ proper $\}$ is infinite, then $\lim _{k \in A}\left\|R_{k}\right\|=\infty$.

That is, a gluing recipe looks like a zoo, except that we allow all or some of the creatures to be just single natural numbers.

Figure 2 shows a zoo $s$ together with a gluing recipe $r$.

3.9. Definition. Let $s=\left(S_{0}, \ldots\right)$ and $t=\left(T_{0}, \ldots\right)$ be zoos, and let $r=\left(R_{0}, R_{1}, \ldots\right)$ be a gluing recipe.

We say that " $t \leq s$ via $r$ " iff there are creatures $S_{k}^{\prime} \leq S_{k}$ with

$$
\bigcup_{n} \operatorname{int}\left(R_{n}\right) \cap \bigcup_{k \in \operatorname{ext}(r)} \operatorname{int}\left(S_{k}^{\prime}\right)=\emptyset
$$

such that $t$ is obtained from $r$ by replacing each $k \in \operatorname{ext}(r)$ by $S_{k}^{\prime}$, i.e.,

$$
T_{n}=\left(R_{n} \backslash \operatorname{ext}\left(R_{n}\right)\right) \cup \underset{k \in \operatorname{ext}\left(R_{n}\right)}{\cup} S_{k}^{\prime},
$$

and $T_{n}^{[\eta]}=S_{k}^{\prime}{ }^{[\eta]}$ for $\eta \in S_{k}^{\prime}, k \in \operatorname{ext}\left(R_{n}\right)$, and $T_{n}^{[\eta]}=\operatorname{int}\left(R_{n}^{[\eta]}\right) \cup \bigcup_{k \in \operatorname{ext}\left(R_{n}^{[\eta]}\right)} S_{k}^{\prime}$ for $\eta \in \operatorname{int}\left(R_{n}\right)$.

Figure 3 shows the zoo $t$ obtained from the zoo $s$ via the gluing recipe $r$ from Figure 2 .

We say " $t \leq s$ " iff there is a gluing recipe $r$ such that " $t \leq s$ via $r$ " holds.

We say " $t \leq^{*} s$ " iff there is some $t^{\prime}={ }^{*} t$ such that $t^{\prime} \leq s$. 

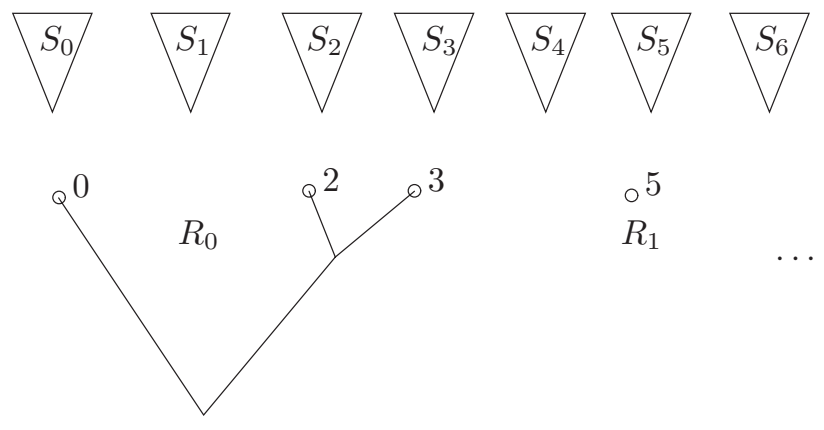

$0^{5}$

$R_{1}$

FiguRe 2.

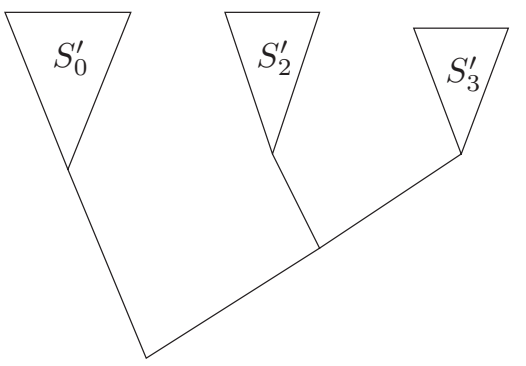

$T_{0}$

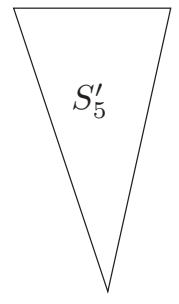

$T_{1}$

FiguRE 3.

We leave it to the reader to check that the relation $t \leq s$ and $t \leq^{*} s$ are indeed transitive (and reflexive). Also, $\leq$ is antisymmetric, and

$$
s \leq^{*} t \& t \leq^{*} s \Rightarrow s={ }^{*} t .
$$

For visualizing creatures, and also for avoiding notational complications, it is often useful to replace the relation $\leq$ by the following relation $\leqq$ :

3.10. Convention. We will write $t \leqq s$ if $t \leq s$ via some gluing recipe $r$, and in addition to

$$
\bigcup_{n} \operatorname{int}\left(R_{n}\right) \cap \bigcup_{k \in \operatorname{ext}(r)} \operatorname{int}\left(S_{k}^{\prime}\right)=\emptyset
$$

we have moreover

$$
\bigcup_{n} \operatorname{int}\left(R_{n}\right) \cap \bigcup_{n} \operatorname{int}\left(S_{n}\right)=\emptyset,
$$

i.e., the internal nodes from $s$ that were omitted (either in the passage from $S_{n}$ to $S_{n}^{\prime}$, or because they are in some $S_{k}$ with $\left.k \notin \operatorname{ext}(r)\right)$ will not be recycled as nodes from $r$. Note that:

(1) The relation $\leqq$ is not transitive.

(2) However: For any $t \leq s$ we can find (by renaming internal nodes of $t$ ) a zoo $t^{\prime} \leqq s, t^{\prime}=\left(T_{0}^{\prime}, \ldots\right)$ such that $t^{\prime}$ is isomorphic to $t$, i.e., there is a bijection between $\bigcup_{n} T_{n}^{\prime}$ and $\bigcup_{n} T_{n}$ which is the identity on leaves, and preserves the relations $\unlhd$ and the norms. 
(3) Moreover: Let $\left(s_{i}: i \in I\right)$ be a family of zoos, and $\forall i: t \leqq s_{i}$. Assume $t_{1} \leq t$ via $r_{1}$. Then there is a gluing recipe $r_{1}^{\prime}$ which is isomorphic to $r_{1}$ and a condition $t_{1}^{\prime}$ isomorphic to $t_{1}$, with $t_{1} \leqq t$ via $r_{1}^{\prime}$, and also satisfying $\forall i: t_{1}^{\prime} \leqq s_{i}$.

Thus, whenever we consider conditions $t \leq s$ we will usually assume without loss of generality that we have even $t \leqq s$. This guarantees that any $\eta \in t \cap s$ will appear in $t$ "in the same place" as in $s$, e.g., $\operatorname{succ}_{t}(\eta) \subseteq \operatorname{succ}_{s}(\eta)$.

We write $t \leqq * s$ iff there is $t^{\prime}=^{*} t$ with $t^{\prime} \leqq s$.

3.11. Definition. Let $E$ be a finite set, and let $s$ be a zoo. A coloring (of $s$, with colors in $E$ ) is a partial map $c: s \rightarrow E$, or $c:[s]^{2} \rightarrow E$, or $c: \operatorname{ext}(s) \rightarrow E$, such that each map $c\left\lceil S_{n}\right.$, or $c \uparrow\left[S_{n}\right]^{2}$, or $c\left\lceil\operatorname{ext}\left(S_{n}\right)\right.$ is a coloring as in 2.7. Again we call $c$ a unary node coloring, a binary node coloring, or a branch coloring, respectively.

Let $t \leqq s$. Then $c$ induces a coloring of $t$, which we also call $c$.

We say that $t$ is $c$-homogeneous if:

- In the first case: for all $n, c\left\lceil\operatorname{succ}_{T_{n}}(\eta)\right.$ is constant, for all $\eta \in \operatorname{int}\left(T_{n}\right) \cap s$.

- In the second case: for all $n, c \uparrow\left[\operatorname{succ}_{T_{n}}(\eta)\right]^{2}$ is constant, for all $\eta \in \operatorname{int}\left(T_{n}\right) \cap$ $s$.

- In the third case: not only is each $c\left\lceil\operatorname{ext}\left(T_{n}\right)\right.$ constant, but all constant values are the same, i.e., $c\lceil\operatorname{ext}(t)$ is constant.

We say that $t \leqq * s$ is almost $c$-homogeneous iff there is $t^{\prime}=^{*} t$, such that $t^{\prime}$ is $c$-homogeneous.

3.12. Fact. If $t$ is $c$-homogeneous, then any $t^{\prime} \leqq t$ is also $c$-homogeneous.

If $t$ is almost $c$-homogeneous, then any $t^{\prime} \leqq^{*} t$ is also almost $c$-homogeneous.

3.13. Lemma. Let $s=\left(S_{0}, \ldots\right)$ be a zoo, and let $E$ be a finite set. Let $c: \bigcup_{n} S_{n} \rightarrow$ $E$, or $c: \bigcup_{n}\left[S_{n}\right]^{2} \rightarrow E$, or $c: \bigcup_{n} \operatorname{ext}\left(S_{n}\right) \rightarrow E$ be a coloring of $s$ with colors from $E$.

Then there is a zoo $t \leqq s$ which is homogeneous for $c$.

Moreover, $t$ can be obtained from s by combining the steps "shrinking" and "dropping" (i.e., with gluing recipes which contain only improper creatures).

Proof. We show this only for the case $|E|=2$. (For larger $E$, repeat the proof $\left\lceil\log _{2}|E|\right\rceil$ many times, or use the unproved assertion from Lemma 2.8.)

We may assume $\left\|S_{n}\right\| \geq \mathcal{R}(4)$ for all $n$. By Ramsey's theorem we can find a sequence $\left(\ell_{n}: n \in \mathbb{N}\right)$ [namely: $\left.\ell_{n}:=\mathcal{R}^{-1}\left(\left\|S_{n}\right\|\right)\right]$ which diverges to infinity and satisfies $\forall n:\left\|S_{n}\right\| \geq \mathcal{R}\left(\ell_{n}\right)$, and $\ell_{n} \geq 4$.

Apply Lemma 2.8 to each $S_{n}$ separately to get creatures $T_{n} \leq S_{n}$ which are homogeneous for $c$ and satisfy $\left\|T_{n}\right\| \geq \ell_{n}$. Thus $t=\left(T_{0}, T_{1}, \ldots\right)$ is a zoo.

If $c$ is a coloring of the third kind (a unary branch coloring), then it is still possible that the constant values that $c$ takes on each creature are different. One of the constant values appears infinitely often, so by dropping creatures from $t$ we obtain $t^{\prime} \leqq t$ which is $c$-homogeneous.

It is clear that we can extend this lemma to the slightly more general case of finitely many colorings: If $s=\left(S_{0}, \ldots\right)$ is a zoo, and $c_{1}, \ldots, c_{k}$ are colorings of $s$, each with finitely many colors $E_{1}, \ldots, E_{k}$, respectively, then there is a zoo $t \leqq s$ which is homogeneous for each $c_{i}$. 
If we have countably many colorings, then we can in general not find a zoo which is homogeneous for all of them; however, the following construction shows that this is almost possible.

3.14. Lemma. Let $\left(s_{0}, s_{1}, \ldots\right)$ be a sequence of zoos with $\forall k, n: k<n \Rightarrow s_{n} \varliminf^{*} s_{k}$. Then there is a zoo $t$ with $\forall n: t \leqq * s_{n}$.

Proof. Let $s_{n}=\left(S_{0}^{n}, S_{1}^{n}, \ldots\right)$. Without loss of generality, we may assume (omitting finitely many creatures from $s_{n}$ if necessary) that we have in fact $s_{n} \leqq s_{k}$ for all $k<n$.

Moreover, we may assume, for all $n$ :

(a) $\min \operatorname{ext}\left(S_{0}^{n+1}\right)>\max \operatorname{ext}\left(S_{0}^{n}\right)$, and

(b) $\left\|S_{0}^{n}\right\| \geq n+4$.

Now let $t:=\left(S_{0}^{0}, S_{0}^{1}, S_{0}^{2}, \ldots\right)$, then we claim that $t \leqq * s_{n}$ for all $n$.

First, note that $t$ is indeed a zoo, by (a) and (b).

We will show only $t \leqq s_{0}$; a similar proof establishing $\left(S_{0}^{n}, S_{0}^{n+1}, \ldots\right) \leqq s_{n}$ is left to the reader.

Write $S_{n}$ for $S_{n}^{0}$.

Since $s_{n}=\left(S_{0}^{n}, S_{1}^{n}, \ldots\right) \leqq s_{0}$, there is a creature $R_{n}$ such that $S_{0}^{n}=\operatorname{int}\left(R_{n}\right) \cup$ $\bigcup_{k \in \operatorname{ext}\left(R_{n}\right)} S_{k}^{\prime}$, where $S_{k}^{\prime} \leq S_{k}$. Note $\left\|R_{n}\right\| \geq\left\|S_{0}^{n}\right\| \geq n$ (if $R_{n}$ is proper).

We leave it to the reader to check that $\max \operatorname{ext}\left(R_{n}\right)<\min \operatorname{ext}\left(R_{n+1}\right)$ (using $\left.\max \operatorname{ext}\left(S_{0}^{n}\right)<\min \operatorname{ext}\left(S_{0}^{n+1}\right)\right)$.

Now the gluing recipe $r=\left(R_{0}, R_{1}, \ldots\right)$ witnesses $t \leqq s_{0}$.

The following corollary will be used in our transfinite construction 5.6 See also 6.1 .

3.15. Corollary. Let $Z$ be a countable set of zoos which is linearly quasiordered by $\varliminf^{*}$. Then there is a zoo $t$ with $\forall s \in Z: t \leqq * s$.

Proof. Let $Z=\left\{z_{0}, z_{1}, \ldots\right\}$. Choose a sequence $s_{0} \geqq * s_{1} \geqq * \ldots$ in $Z$ such that $s_{n} \in Z$, and $s_{n} \leqq{ }^{*} z_{n}$, and apply Lemma 3.14 .

3.16. Corollary. Let $s=\left(S_{0}, \ldots\right)$ be a zoo, and for each $n \in \mathbb{N}$ let $c_{n}: s \rightarrow E_{n}$ or $c_{n}:[s]^{2} \rightarrow E_{n}$ or $c_{n}: \operatorname{ext}(s) \rightarrow E_{n}$ be a coloring with finitely many colors.

Then there is a zoo $t \leqq *$ which is almost homogeneous for each $c_{n}$. That is, there is a zoo $t \leqq{ }^{*} s$, and for each $n$ there is $t_{n}={ }^{*} t, t_{n} \leqq s$, which is homogeneous for $c_{n}$.

Proof. Apply Lemma 3.13 infinitely many times to get a decreasing sequence $s \geqq$ $s_{0} \geqq s_{1} \geqq \cdots$ such that each $s_{i}$ is $c_{i}$-homogeneous. By 3.14 there is a $\leqq *$-lower bound $t$ for this sequence; by $3.12 t$ is almost $c_{i}$-homogeneous for each $i$.

\section{Gauging growth}

4.1. Motivation. The comparison " $f \leq g$ " between growth functions is too coarse for our purposes. For finer comparisons, we will consider the growth behavior of these functions "locally". For example, if $I=[a, b]$ is an interval in $\mathbb{N}$, and $f(I) \cap I=\emptyset$ (i.e., $f(a)>b$ ), then we can say that $f$ grows fast on $I$, or symbolically: $f\left\lceil I>I\right.$. Similarly, if $\mathcal{I}=\left\{\left[a_{1}, b_{1}\right], \ldots,\left[a_{n}, b_{n}\right]\right\}$ is a set of intervals with $a_{1}<b_{1}<$ $\cdots<a_{n}<b_{n}$, and $f\left(a_{1}\right)>b_{n}$, we can say that $f$ "grows faster than $\mathcal{I}$ ", or "is stronger than the set $\mathcal{I}$ ", symbolically: $f\lceil\mathcal{I}>\mathcal{I}$. 
This point of view allows us to introduce a dual concept: If again we have $a_{1}<b_{1}<\cdots<a_{n}<b_{n}$, but now $f\left(b_{k}\right)<a_{k+1}$ for $k=1, \ldots, n-1$, then we can say that $f$ "grows more slowly than $\mathcal{I}$ ", or $f$ is "weaker" than the set $\mathcal{I}$, symbolically: $f \mid \mathcal{I}<\mathcal{I}$.

Note that this is indeed a local notion: If $\mathcal{I}_{1}$ and $\mathcal{I}_{2}$ are sets of intervals, $f$ and $g$ growth functions, then it is quite possible that $f \mid \mathcal{I}_{1}<\mathcal{I}_{1}$ but $f \mid \mathcal{I}_{2}>\mathcal{I}_{2}$, while $g$ satisfies the converse inequalities.

How does this help us to compare $f$ and $g$ ? If we label certain sequences of intervals as "distinguished", we introduce a (kind of) ordering relation on growth functions: $f<g$ iff there is a distinguished sequence of intervals $\mathcal{I}$ which is stronger than $f$ but weaker than $g: f \nmid \mathcal{I}<\mathcal{I}<g \mid \mathcal{I}$. Our aim in the remaining sections is to show how we can select such distinguished sequences such that the resulting ordering relations can be viewed as a linear order.

Zoos (or rather: nodes in zoos) are our way of coordinatizing sequences of intervals. If $s$ is a zoo, $\eta \in s$, then $\eta$ is associated with the interval $[\min [\eta], \max [\eta]]$, and also with the set of intervals $\{[\min [\nu], \max [\nu]]: \nu \in \operatorname{succ}(\eta)\}$.

4.2. Definition. Let $f$ be a growth function, and $s=\left(S_{0}, S_{1}, \ldots\right)$ a zoo.

(a) $s$ is $f$-strong iff

$$
\forall n: \quad f\left(\max \operatorname{ext}\left(S_{n}\right)\right) \leq \min \operatorname{ext}\left(S_{n+1}\right) .
$$

Not surprisingly, we say that $s$ is almost $f$-strong if the above inequality holds for all but finitely many $n$.

(b) Let $\eta \in \operatorname{int}\left(S_{n}\right)$. We call $\eta f$-strong in $s$ iff for all $\nu_{1}<\nu_{2}$ in $\operatorname{succ}_{S_{n}}(\eta): f\left(\max \left[\nu_{1}\right]\right) \leq \min \left[\nu_{2}\right]$.

(c) Let $\eta \in S_{n}$. We call $\eta f$-weak in $s$ iff $f(\min [\eta])>\max [\eta]$.

\subsection{Fact.}

(1) No $\eta$ can be both weak and strong.

(2) For $\eta \in \operatorname{ext}\left(S_{n}\right), \max [\eta]=\min [\eta]=\eta \in \mathbb{N}$. So (since $f$ is a growth function), every leaf is $f$-weak: $f(\eta)>\eta$.

(3) If $\eta \unlhd \nu$, and $\eta$ is weak, then $\nu$ cannot be strong.

(4) If $t \leqq s$, and $\eta \in t \cap s$ is $f$-weak in $s$, then $\eta$ is also $f$-weak in $t$.

(5) Similarly, if $\eta$ is $f$-strong in $s$, then $\eta$ is also $f$-strong in $t$.

(6) Finally, if $s$ is $f$-strong, then any $t \leqq s$ is also $f$-strong.

4.4. Fact. Let $s$ be a zoo, $f$ a growth function. Then there is $t \leqq s$ which is $f$-strong.

Proof. The sequence $\left(\min \operatorname{ext}\left(S_{n}\right): n=0,1,2, \ldots\right)$ diverges to infinity, so replacing $\left(S_{0}, S_{1}, \ldots\right)$ by a subsequence, if necessary, we get $f\left(\max \operatorname{ext}\left(S_{n}\right)\right)<\min \operatorname{ext}\left(S_{n+1}\right)$ for all $n$.

4.5. Definition. Let $s$ be a zoo, $f$ a growth function, $F \subseteq s$ a *front.

We say that $F$ gauges $f$ (or more precisely: $F$ gauges $f$ in $s$ ) iff

(1) all $\eta \in F$ are $f$-weak,

(2) whenever $\eta_{1}<\eta_{2}$ are in $F$, then $f\left(\max \left[\eta_{1}\right]\right) \leq \min \left[\eta_{2}\right]$ (since $f$ is monotone, it is enough to require this only for all $\left.\eta_{1} \lessdot \eta_{2}\right)$,

(3) $s$ is almost $f$-strong. (This actually follows from (2).) 
We say that $s$ gauges $f$ iff there is a ${ }^{*}$ front $F \subseteq s$ which gauges $f$. In this case we fix such a ${ }^{*}$ front (or, if possible, an actual front) and call it $F(s, f)$.

The following lemma is easy but important:

\subsection{Crucial Lemma.}

(1) If $F_{1}, F_{2} \subseteq s$ both gauge $f$, then $F_{1} \approx^{*} F_{2}$.

(2) If $s$ gauges $f$, and $t \leqq * s$, then also $t$ gauges $f$, and $F(t, f) \approx^{*} F(s, f) \cap t$.

Proof. (1) Let $s=\left(S_{0}, S_{1}, \ldots\right)$. Without loss of generality (dropping finitely many creatures if necessary) we may assume that $F_{1}$ and $F_{2}$ are not only *fronts but actually fronts. Now assume $F_{1} \neq F_{2}$. So there is (wlog) $\eta \in \operatorname{int}\left(S_{n}\right) \cap\left(F_{1} \backslash F_{2}\right)$ such that $F_{2} \cap S_{n}^{[\eta]}$ is a front in $S_{n}^{[\eta]}$. Let $\nu_{1}<\nu_{2}$ be in $F_{2} \cap S_{n}^{[\eta]}$. Since $F_{2}$ gauges $f$ we must have $f\left(\max \left[\nu_{1}\right]\right) \leq \min \left[\nu_{2}\right]$, but since $\eta$ is $f$-weak we must also have $f(\min [\eta])>\max [\eta]$. Clearly $\min [\eta] \leq \min \left[\nu_{1}\right]$ and $\max [\eta] \geq \max \left[\nu_{2}\right]$, so we have a contradiction.

(2) Let $F^{\prime}:=F(s, f) \cap t$. Clearly $F^{\prime}$ is a *front in $t$, and $F^{\prime}$ gauges $f$, so $F^{\prime} \approx^{*} F(t, f)$ by $(1)$.

The following fact is easy:

4.7. Fact. Let $F$ be a front in $s$. Then there is a growth function $f$ and $t \leqq s$ such that $t$ gauges $f$, and $F(t, f) \approx^{*} F \cap t$. (In fact, we can choose $t=s$.)

Proof. Choose a growth function $f$ satisfying $f(\min [\eta])=f(\max [\eta])=\max [\eta]+1$ for all $\eta \in F$. We leave the details to the reader.

4.8. Lemma. Let $f$ be a growth function, and $s=\left(S_{0}, S_{1}, \ldots\right)$ a zoo which is almost $f$-strong. Then there is $t \leqq s$ such that $t$ gauges $f$.

In fact, we will find a coloring function $c$ such that any $t \leqq s$ which is almost c-homogeneous will gauge $f$, and then invoke 3.13 to show that there is such $t$.

Proof. We start with a zoo $s=\left(S_{0}, S_{1}, \ldots\right)$ which is $f$-strong.

By ignoring finitely many of the $S_{i}$ we may assume $\left\|S_{n}\right\| \geq \mathcal{R}(\mathcal{R}(4))$ for all $n$.

For each $\eta \in \operatorname{int}(s)$ we define a pair coloring $c_{\eta}$ of $\operatorname{succ}_{s}(\eta)$ with three colors as follows: Whenever $\nu<\nu^{\prime}$ in $\operatorname{succ}_{s}(\eta)$, then

- $c_{\eta}\left(\left\{\nu, \nu^{\prime}\right\}\right)=$ strong, if $f(\max [\nu])<\min \left[\nu^{\prime}\right]$,

- $c_{\eta}\left(\left\{\nu, \nu^{\prime}\right\}\right)=$ weak, if $f(\min [\nu])>\max \left[\nu^{\prime}\right]$,

- $c_{\eta}\left(\left\{\nu, \nu^{\prime}\right\}\right)=$ undecided, otherwise.

Note that if $\nu_{1}<\nu_{2}<\nu_{3}<\nu_{4}$ are in $\operatorname{succ}_{s}(\eta)$, then at least one of

$$
c\left(\left\{\nu_{1}, \nu_{4}\right\}\right)=\text { strong } \quad \text { or } \quad c\left(\left\{\nu_{2}, \nu_{3}\right\}\right)=\text { weak }
$$

has to hold, since otherwise we would have

$$
f\left(\max \left[\nu_{1}\right]\right) \geq \min \left[\nu_{4}\right] \text { and } f\left(\min \left[\nu_{2}\right]\right) \leq \max \left[\nu_{3}\right],
$$

which together with

$\min \left[\nu_{1}\right] \leq \max \left[\nu_{1}\right]<\min \left[\nu_{2}\right] \leq \max \left[\nu_{2}\right]<\min \left[\nu_{3}\right] \leq \max \left[\nu_{3}\right]<\min \left[\nu_{4}\right] \leq \max \left[\nu_{4}\right]$ yields a contradiction to the fact that $f$ is monotone.

(Also note that if $f\left(\max \operatorname{ext}\left(S^{[\nu]}\right)\right)<\min \operatorname{ext}\left(S^{\left[\nu^{\prime}\right]}\right)$, and $T \leq S$ with $\nu, \nu^{\prime} \in T$, then we also have $f\left(\max \operatorname{ext}\left(T^{[\nu]}\right)\right)<\min \operatorname{ext}\left(T^{\left[\nu^{\prime}\right]}\right)$.)

The family $\left(c_{\eta}: \eta \in \operatorname{int}(s)\right)$ defines on $s$ a binary node coloring $c$. Let $t \leqq s$ be $c$-homogeneous, $t=\left(T_{0}, T_{1}, \ldots\right),\left\|T_{n}\right\| \geq 4$ for all $n$. 


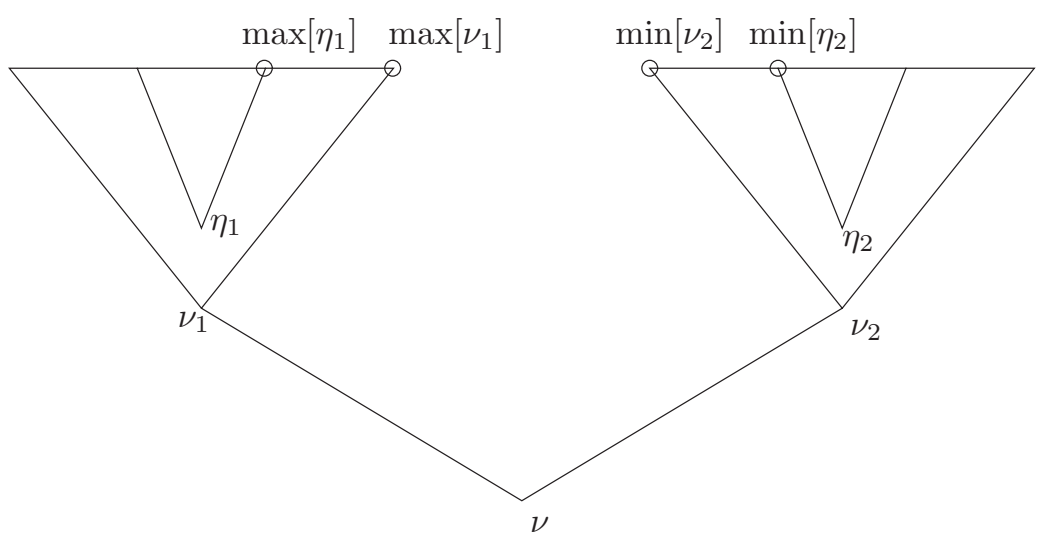

FIGURE 4.

Since each set $\operatorname{succ}_{T_{n}}(\eta)$ has more than 3 elements, it is impossible that $c_{\eta}$ is constantly "undecided".

Clearly each $\eta \in t$ is either $f$-weak or $f$-strong.

Now we show that any $c$-homogeneous zoo $t \leqq s$ gauges $f$. (Note that if $t \leqq s$ via $r$, then the $f$-strength of $s$ ensures that almost all $\eta \in \operatorname{int}(r)$ will be $f$-strong in $t$.)

On every branch $b$ let $\eta_{b}$ be the $\triangleleft$-lowest node on $b$ which is $f$-weak (recall that all leaves are $f$-weak), and let $F:=F(t, f):=\left\{\eta_{b}: b\right.$ a branch in $\left.t\right\}$.

Note that if $\nu \unrhd \eta_{b}$, then $\nu$ is $f$-weak (by Fact 4.3), while any $\nu \triangleleft \eta_{b}$ is $f$-strong.

So $F \cap b=\left\{\eta_{b}\right\}$ for all $b$. Hence $F(t, f)$ is a front.

Let $\eta_{1}<\eta_{2}$ be in $F, \eta_{1} \in T_{n_{1}}, \eta_{2} \in T_{n_{2}}$. We have to check that $f\left(\max \left[\eta_{1}\right]\right) \leq$ $\min \left[\eta_{2}\right]$.

The case $n_{1}<n_{2}$ is trivial (since $t$ is $f$-strong).

So assume $n_{1}=n_{2}=: n$. In $\left(T_{n}, \unlhd\right)$ let $\nu$ be the greatest lower bound of $\eta_{1}$ and $\eta_{2}$. Then $\nu \triangleleft \eta_{1}$, so $\nu$ is $f$-strong. Let $\nu_{1}<\nu_{2}$ in $\operatorname{succ}(\nu), \nu_{1} \unlhd \eta_{1}, \nu_{2} \unlhd \eta_{2}$; see Figure 4.

Clearly $f\left(\max \left[\eta_{1}\right]\right) \leq f\left(\max \left[\nu_{1}\right]\right) \leq \min \left[\nu_{2}\right] \leq \min \left[\eta_{2}\right]$ (where the middle inequality holds because $\nu$ is $f$-strong).

Hence $F$ gauges $f$.

We now fix a zoo $s$; the fronts in $s$ are naturally partially ordered by the relation "is everywhere higher". We will show below that the relation $f \leq_{\operatorname{ext}(s)} g$ (see 1.13(2)) can be translated to a " $F(s, f)$ is higher than $F(s, g)$ ", for sufficiently small $s$.

4.9. Definition. Let $s$ be a zoo, and let $F$ and $G$ be ${ }^{*}$ fronts in $s$. We write $F \prec G$ (or $F \prec{ }_{s} G$ ) iff, for all branches $b$ of $s, b$ meets $F \triangleleft$-above $G$, i.e., letting $b \cap F=\left\{\eta_{F, b}\right\}, b \cap G=\left\{\eta_{G, b}\right\}$, we have $\eta_{F, b} \triangleright \eta_{G, b}$. (The reason for this apparent reversal of inequalities will become clear in Remark 4.14 below.)

Recall that $F \approx^{*} G$ iff there is some $s^{\prime}={ }^{*} s$ such that $F \cap s^{\prime}=G \cap s^{\prime}$.

We write $F \prec_{s}^{*} G$ if the relation $\eta_{F, b} \triangleright \eta_{G, b}$ holds for almost all branches $b$ of $s$ (i.e., for all except finitely many). Equivalently, $F \prec_{s}^{*} G$ iff there is some $s^{\prime}={ }^{*} s$ such that $F \cap s^{\prime} \prec_{s^{\prime}} G \cap s^{\prime}$. 
Similarly, we define $F \preccurlyeq_{s} G\left[F \preccurlyeq_{s}^{*} G\right]$, by requiring that for all [except finitely many] branches $b, b$ meets $F \unlhd$-above $G$, i.e., letting $b \cap F=\left\{\eta_{F, b}\right\}, b \cap G=\left\{\eta_{G, b}\right\}$, we have $\eta_{F, b} \unrhd \eta_{G, b}$.

We write $F+1 \approx_{s}^{*} G$ iff $F \prec_{s}^{*} G$, and moreover, for almost all branches $b$ of $s$, $\eta_{F, b}$ is a direct successor of $\eta_{G, b}$.

Similarly, we write $F+n \approx_{s}^{*} G$ iff $F \preccurlyeq_{s}^{*} G$, and moreover, for almost all branches $b$ of $s, \eta_{F, b}$ is exactly $n$ nodes above $\eta_{G, b}$.

The notations $F+n \preccurlyeq_{s}^{*} G$ and $G \preccurlyeq_{s}^{*} F+n$ have the obvious meanings. In particular, $F+1 \preccurlyeq_{s}^{*} G$ will be equivalent to $F \prec_{s}^{*} G$.

Finally, we write $F+\infty \preccurlyeq_{s}^{*} G$ iff for all $n$, for almost all branches $b$ of $s, \eta_{F, b}$ is more than $n$ nodes above $\eta_{G, b}$. (Equivalently, if for all $n, F+n \preccurlyeq_{s}^{*} G$.)

4.10. Remark.

(1) It is easy to see that $\left[F+n \approx^{*} G\right.$ and $\left.F+n \approx^{*} G^{\prime}\right]$ implies $G \approx^{*} G^{\prime}$, so this functional notation is justified.

(2) There are fronts $F$ such that $F+1$ is undefined. For example, if we let $F$ be the front

$$
\operatorname{root}(s):=\left\{\operatorname{root}\left(S_{0}\right), \operatorname{root}\left(S_{1}\right), \ldots\right\},
$$

then there is no *front $G$ with $F+1 \approx_{s}^{*} G$.

(3) However, if $F+1 \preccurlyeq_{s}^{*} \operatorname{root}(s)$, then $F+1$ is "well defined", i.e., there is a *front $G$ with $F+1 \approx_{s}^{*} G$, and by (1), $G$ is unique up to $\approx_{s}^{*}$.

4.11. Fact. If $F \preccurlyeq_{s}^{*} G$, and $t \leqq^{*} s$, then also $(F \cap t) \preccurlyeq_{t}^{*}(G \cap t)$. Similarly, for $F+n \preccurlyeq_{s}^{*} G$ or $F+\infty \preccurlyeq_{s}^{*} G$.

\subsection{Lemma.}

(1) Let $s$ be a zoo, and let $F, G$ be *fronts in $s$. Then there is a coloring $c$ such that whenever $t \leqq^{*} s$ is almost c-homogeneous, then $(F \cap t) \preccurlyeq_{t}^{*}(G \cap t)$ or $(G \cap t) \preccurlyeq_{t}^{*}(F \cap t)$.

(2) Let $s$ be a zoo, and let $F \preccurlyeq_{s}^{*} G$ be *fronts in $s$. Then there is a coloring $c$ of $s$ such that whenever $t \leqq^{*} s$ is almost c-homogeneous, then exactly one of the following holds:

- $(F \cap t) \approx_{t}^{*}(G \cap t)$,

- $(F \cap t)+1 \preccurlyeq_{t}^{*}(G \cap t)$.

(3) Let $s$ be a zoo, and let $F \preccurlyeq_{s}^{*} G$ be *fronts in $s$. Then there are colorings $c_{0}, c_{1}, \ldots$ such that whenever $t \leqq s$ is almost $c_{k}$-homogeneous for all $k$, then exactly one of the following holds:

- $(F \cap t)+n \approx_{t}^{*}(G \cap t)$ for some (unique) $n$,

- $(F \cap t)+\infty \preccurlyeq_{t}^{*}(G \cap t)$.

Proof. (1) For each branch $b$ of $s$ that meets $F$ ( $G$, respectively) in a unique point, let $\left\{\eta_{F, b}\right\}=F \cap b\left(\left\{\eta_{G, b}\right\}=G \cap b\right.$, respectively).

Now color each branch $b$ as follows:

- $c(b)=\operatorname{small}$ if $\eta_{F, b} \unrhd \eta_{G, b}$,

- $c(b)=$ big if $\eta_{F, b} \triangleleft \eta_{G, b}$,

- $c(b)=$ unknown if $\eta_{F, b}$ and/or $\eta_{G, b}$ is undefined.

Now let $t \leqq * s$ be almost homogeneous for $c$. Clearly the color unknown appears only finitely many times as a value of $c\lceil\operatorname{ext}(t)$. If $c\lceil\operatorname{ext}(t)$ is almost constant with value small, then $(F \cap t) \preccurlyeq *(G \cap t)$, otherwise $(G \cap t) \preccurlyeq *(F \cap t)$. 
(2) Define $\eta_{F, b}$ and $\eta_{G, b}$ as above. Define a branch coloring $c$ as follows:

- $c(b)=$ equal if $\eta_{F, b}=\eta_{G, b}$,

- $c(b)=$ bigger if $\eta_{F, b} \triangleright \eta_{G, b}$,

- $c(b)=$ unknown if $\eta_{F, b}$ and/or $\eta_{G, b}$ is undefined, or if $\eta_{F, b} \triangleleft \eta_{G, b}$.

Again, any almost homogeneous condition can take the value unknown at most finitely many times.

(3) For each $n=0,1,2, \ldots$ define a branch coloring $c_{n}$ as follows:

- $c_{n}(b)=$ small if $\eta_{F, b}$ is at most $n$ nodes $\triangleleft$-above $\eta_{G, b}$,

- $c_{n}(b)=$ big if $\eta_{F, b}$ is more than $n$ nodes above $\eta_{G, b}$,

- $c_{n}(b)=$ unknown if $\eta_{F, b}$ and/or $\eta_{G, b}$ is undefined, or if $\eta_{F, b} \triangleleft \eta_{G, b}$.

Now assume that $t \leqq{ }^{*} s$ is almost $c_{n}$-homogeneous, for all $n$. We distinguish two cases:

(Case 1) There is some $n$ such that $c_{n}\lceil\operatorname{ext}(t)$ is constantly small (with finitely many exceptions). Let $\bar{n}$ be the smallest $n$ for which this happens, then on almost all branches $b$ of $t, \eta_{F, b}$ is exactly $\bar{n}$ nodes above $\eta_{G, b}$, so

$$
(F \cap t)+\bar{n} \approx_{t}^{*}(G \cap t) .
$$

(Case 2) Each $c_{n}$ is (almost equal to) the constant function with value big. Then we can easily see that

$$
(F \cap t)+\infty \preccurlyeq_{t}^{*}(G \cap t) .
$$

4.13. Definition. Let $f$ and $g$ be growth functions, and assume that $s$ gauges both $f$ and $g$. (So $F(s, f)$ and $F(s, g)$ are well defined.)

We now write $f \approx_{s}^{*} g, f \prec_{s}^{*} g$, etc., iff $F(s, f) \approx^{*} F(s, g), F(s, f) \prec_{s}^{*} F(s, g)$, etc., respectively.

Combining 4.6 and 4.11, we get: If $f \prec_{s}^{*} g$, and $t \varliminf^{*} s$, then also $f \prec_{t}^{*} g$, etc.

4.14. Remark. If $f \leq g$, then every $g$-strong node is also $f$-strong, and every $f$-weak node is $g$-weak.

Hence the front corresponding to $f$ is $\unlhd$-higher in the trees than the one for $g$.

Thus, $f \leq g$ implies $f \preccurlyeq_{s}^{*} g$, whenever $s$ gauges $f$ and $g$.

The converse is of course not true, but we will show below that it is "true modulo $\operatorname{ext}(s)$ ":

4.15. Lemma. Let $s$ be $a$ zoo, let $f$ and $g$ be growth functions, and let $s$ gauge $f$ and $g$.

Assume $f \preccurlyeq_{s}^{*} g$.

Then $f \leq \operatorname{ext}(s) g .($ See 1.13.)

Proof. Write $A$ for $\operatorname{ext}(s)$.

First note that if $s={ }^{*} s^{\prime}$, then also $\operatorname{ext}(s)={ }^{*} \operatorname{ext}\left(s^{\prime}\right)$, so by 1.14 we may (replacing $s$ by an appropriate $s^{\prime}={ }^{*} s$ ), without loss of generality, assume that not only $F(f, s) \preccurlyeq_{s}^{*} F(g, s)$ but even $F(f, s) \preccurlyeq s F(g, s)$.

Part 1. We first show $\forall k \in A: f(k) \leq\left(h_{A} \circ g\right)(k)$.

So fix $k \in A$. Let $\eta \in F(f, s), \eta \unlhd k$.

Let $\eta^{\prime} \in F(f, s)$, where $\eta \lessdot \eta^{\prime}$.

So $f(k) \leq f(\max [\eta]) \leq \min \left[\eta^{\prime}\right]$, because $F(s, f)$ gauges $f$. 
But $g(k) \geq g(\min [\eta])>\max [\eta]$, as $\eta$ is $g$-weak. By the definition of $\eta \lessdot \eta^{\prime}$ this means that $h_{A}(g(k)) \geq \min \left[\eta^{\prime}\right]$, as $h_{A}(\cdots) \in A$.

So $f(k) \leq\left(h_{A} \circ g\right)(k)$.

Part 2. We now consider a general $n \in \mathbb{N}$. Let $k:=h_{A}(n)$. Clearly $n \leq k \in A=$ $\operatorname{ext}(s)$. So by part $1, f(n) \leq f(k) \leq\left(h_{A} \circ g\right)(k)=\left(h_{A} \circ g \circ h_{A}\right)(n)$.

So in any case we have $f \leq h_{A} \circ g \circ h_{A}$, which means $f \leq_{A} g$.

4.16. Lemma. If $f \prec_{s}^{*} g$, then $f \leq \operatorname{ext}(s) g$ and $g \not \leq_{\operatorname{ext}(s)} f$.

Proof. We already have $f \leq \operatorname{ext}(s) g$, so we only have to refute $g \leq_{\operatorname{ext}(s)} f$.

Again replacing $s$ by an appropriate $s^{\prime}={ }^{*} s$ we may assume $f \prec_{s} g$. For notational simplicity only we will assume that the *fonts $F(s, f)$ and $F(s, g)$ are actually fronts.

Let $A:=\operatorname{ext}(s)$. Assume $g \leq \max \left(h_{A}, f\right)^{(j)}$. All except finitely many $\eta \in \operatorname{int}(s)$ have more than $j$ direct successors; find $n$ and $\eta \in F(s, g) \cap S_{n}$ such that $\eta$ has more than $j$ successors. Consider the set $C:=\{\nu \in F(s, f): \eta \triangleleft \nu\}$. We know that this set is nonempty and even that it has more than $j$ elements (since each branch through $\eta$ must meet $C$ ). We can write $C$ as $C=\left\{\nu_{1}, \ldots, \nu_{\ell}\right\}$, where $\ell>j$ and $\nu_{1} \lessdot \nu_{2} \lessdot \cdots \lessdot \nu_{\ell}$.

Then $h_{A}\left(\min \left[\nu_{i}\right]\right) \leq \min \left[\nu_{i+1}\right]$ for all $i$, and also $f\left(\min \left[\nu_{i}\right]\right) \leq \min \left[\nu_{i+1}\right]$, as $\nu_{i} \in F(s, f)$. Hence

$$
\max \left(h_{A}, f\right)^{(j)}\left(\min \left[\nu_{0}\right]\right) \leq \max \left[\nu_{\ell}\right] .
$$

But $g\left(\min \left[\nu_{0}\right]\right)>\max \left[\nu_{\ell}\right]$, because $\eta$ is $g$-weak (as $\eta \in F(s, g)$ ).

The last two facts allow us to replace the relation $\leq_{A}$ between functions (this is the relation that we are really interested in) by the relation $\preccurlyeq_{s}^{*}$ between the associated fronts (this is the relation that can be more easily manipulated, by modifying s), assuming that $A=\operatorname{ext}(s)$ and that $s$ "knows enough" about $f$ and $g$.

\section{DiRECT Limit}

We will fix a nonempty partial order $(I, \leq)$ in which every countable set has an upper bound. Later we will consider only the special case $I=\omega_{1}$.

5.1. Definition. Let $\vec{s}=\left(s_{i}: i \in I\right)$ be a sequence of zoos with $\forall i<j: s_{j} \leqq{ }^{*} s_{i}$. We say that $F$ is a $*$ front in $\vec{s}$ if there is $i \in I$ such that $F \subseteq s_{i}$ is a *front in $s_{i}$. (Note that this implies that each $F \cap s_{j}$ is a *front in $s_{j}$, for all $j \geq i$.)

5.2. Definition. Let $\vec{s}$ be as above, and let $F$ and $G$ be ${ }^{*}$ fronts in $s_{i_{1}}, s_{i_{2}}$, respectively.

We will write $F \approx_{\vec{s}}^{*} G$ iff there is some $i \geq i_{1}, i_{2}$ such that we have $\left(F \cap s_{i}\right) \approx_{s_{i}}^{*}$ $\left(G \cap s_{i}\right)$. Equivalently, we could demand:

For some $i_{*} \geq i_{1}, i_{2}$, for all $i \geq i_{*}:\left(F \cap s_{i}\right) \approx_{s_{i}}^{*}\left(G \cap s_{i}\right)$.

Clearly, this is an equivalence relation.

Similarly, we define $F \preccurlyeq_{\vec{s}} G, F \preccurlyeq_{\vec{s}}^{*} G, F+n \approx_{\vec{s}}^{*} G$, etc. (See Definition 4.9 and Fact 4.11)

For example, $F+n \approx_{\vec{s}}^{*} G$ iff one or both of the following two equivalent conditions hold:

(1) $\exists i:\left(F \cap s_{i}\right)+n \approx_{s_{i}}^{*}\left(G \cap s_{i}\right)$,

(2) $\exists i_{0} \forall i \geq i_{0}:\left(F \cap s_{i}\right)+n \approx_{s_{i}}^{*}\left(G \cap s_{i}\right)$. 
Given $\vec{s}$, we write $\mathcal{F} \vec{s}$ for the set of $\approx_{\vec{s}^{*}}^{*}$ classes of ${ }^{*}$ fronts. $\mathcal{F} \vec{s}$ is naturally partially ordered by $\preccurlyeq_{\vec{s}}^{*}$.

5.3. Definition. Let $\vec{s}$ be as above.

For each $i$, if $s_{i}=\left(S_{0}^{i}, S_{1}^{i}, \ldots\right)$, we let $\operatorname{root}\left(s_{i}\right)$ be the front

$$
\left\{\operatorname{root}\left(S_{0}^{i}\right), \operatorname{root}\left(S_{1}^{i}\right), \ldots\right\} \text {. }
$$

5.4. Definition. $\vec{s}=\left(s_{i}: i \in I\right)$ is "sufficiently generic" if:

(1) $i<j$ implies $s_{j} \leqq * s_{i}$.

(2) For any $i \in I$, and any coloring $c$ of $s_{i}$ (of one of the three types described in 3.11) there is $j \in I, j>i$, such that $s_{j}$ is almost $c$-homogeneous.

(3) For all growth functions $f: \mathbb{N} \rightarrow \mathbb{N}$ there is $i$ such that $s_{i}$ is almost $f$-strong.

(4) For all $i$ there is $j \geq i$ such that $\left(\operatorname{root}\left(s_{i}\right) \cap s_{j}\right)+1 \preccurlyeq_{s_{j}}^{*} \operatorname{root}\left(s_{j}\right)$.

At the end of this section we will show that (assuming $\mathrm{CH}$ ) there exists a sufficiently generic sequence $\left(s_{i}: i \in \omega_{1}\right)$.

But first we will show how a sufficiently generic sequence helps to get the desired clone. We first show that the set $\mathcal{F} \vec{s}$ of $\approx_{\vec{s}}^{*}$-equivalence classes of ${ }^{*}$ fronts is linearly ordered by $\leq_{\vec{s}}$, without a last element.

As a byproduct, we get more information about this linear order (such as: every element has a direct successor). Assuming $\mathrm{CH}$, this information will be sufficient to characterize this order up to order isomorphism.

We then consider the filter $U$ generated by the sets $\operatorname{ext}\left(s_{i}\right)$. The results from the previous section will easily show that the map $f \mapsto F\left(s_{i}, f\right)$ (for an appropriate $i=i(f) \in I$ ) induces an isomorphism between the order $\mathcal{G} / U$ and $\mathcal{F} \vec{s}$. This is enough to prove our main theorem.

5.5. Fact. Assume that $\vec{s}=\left(s_{i}: i \in I\right)$ is sufficiently generic. Then:

(5) For all $i$ : if $c_{0}, c_{1}, \ldots$ are colorings of $s_{i}$, then there is $j>i$ such that $s_{j}$ is almost $c_{k}$-homogeneous for each $k$.

(6) For all *fronts $F, G$ we have $F \preccurlyeq_{\vec{s}}^{*} G$ or $G \preccurlyeq{ }_{\vec{s}}^{*} F$.

(7) For all *fronts $F, G$ with $F \preccurlyeq_{\vec{s}}^{*} G$, exactly one of the following holds:

- $F+n \approx_{\vec{s}}^{*} G$ for some (unique) $n$,

- $F+\infty \preccurlyeq_{\vec{s}}^{*} G$.

(8) For all *fronts $F$ there is a $*$ front $G$ such that $F+1 \approx_{\vec{s}}^{*} G$.

(9) Every countable set of *fronts has a $\preccurlyeq{ }_{s}^{*}$-upper bound. Moreover, if $\mathscr{F}_{1}$ and $\mathscr{F}_{2}$ are countable sets of ${ }^{*}$ fronts and $\forall F_{1} \in \mathscr{F}_{1} \forall F_{2} \in \mathscr{F}_{2}: F_{1} \preccurlyeq_{\vec{s}}^{*} F_{2}$, then there is a $*$ front $G$ with

$$
\forall F_{1} \in \mathscr{F}_{1} \forall F_{2} \in \mathscr{F}_{2}: F_{1} \preccurlyeq_{\vec{s}}^{*} G \preccurlyeq_{\vec{s}}^{*} F_{2} .
$$

(10) For all *fronts $F$ there is a *front $G$ such that $F+\infty \preccurlyeq_{\vec{s}}^{*} G$.

(11) Whenever $F+\infty \preccurlyeq_{\vec{s}}^{*} G$, then there are $2^{\aleph_{0}}$ equivalence classes of ${ }^{*}$ fronts between $F$ and $G$. (Hence by (7) and (10), if we divide the set $\mathcal{F} \vec{s}$ by the equivalence relation generated by "the interval $[x, y]$ is finite", then we get a linear order which is $2^{\aleph_{0}}$-dense.)

(12) For all $i, j: \operatorname{ext}\left(s_{i}\right) \approx_{\vec{s}}^{*} \operatorname{ext}\left(s_{j}\right)$; the equivalence class of these ${ }^{*}$ fronts is the $\preccurlyeq{ }_{s}^{*}$-smallest class.

(13) For all *fronts $G$ not in the class described in (12), there is a *front $F$ with $F+1 \approx_{\vec{s}}^{*} G$.

(14) For all $f: \mathbb{N} \rightarrow \mathbb{N}$ there is $i$ such that $s_{i}$ gauges $f$. 
Proof.

(5) We can find a sequence $i \leq i_{0} \leq i_{1} \leq \cdots$ in $I$ such that $s_{i_{n}}$ is almost $c_{n^{-}}$ homogeneous. Now let $j$ be any upper bound of the set $\left\{i_{0}, i_{1}, \ldots\right\}$, then $s_{j}$ must be almost $c_{n}$-homogeneous for all $n$.

(6) By $4.12(1)$, and $5.4(1,2)$.

(7) By (5) and 4.12(3).

(8) Let $F$ be a ${ }^{*}$ front in $s_{i}$. First we show that there is a ${ }^{*}$ front $H$ such that $F+1 \preccurlyeq_{\vec{s}}^{*} H$. If $\operatorname{root}\left(s_{i}\right)$ cannot serve as such an $H$, then we must have $F \approx_{s_{i}}^{*} \operatorname{root}\left(s_{i}\right)$, so we can use 5.4 (4) to get an appropriate $H$.

Now use 4.10 (3).

(9) Not needed for our main conclusion, and left to the reader.

(10) Use (8) and (9).

(11) Not needed for our main conclusion, and left to the reader.

(12) Clear.

(13) Clear.

(14) Use 4.8 and 5.4 $(1,2,3)$.

The following transfinite construction is now routine.

5.6. Conclusion. Assume CH. Then there is a sufficiently generic sequence $\left(s_{i}\right.$ : $\left.i \in \omega_{1}\right)$.

Proof. Recall that $\omega_{1}$ is an uncountable well-ordered set with the property that for all $j \in \omega_{1}$ the set $\left\{i \in \omega_{1}: i<j\right\}$ is countable. Also recall that each element $i \in \omega_{1}$ has a direct successor

$$
i+1:=\min \left\{j \in \omega_{1}: i<j\right\} .
$$

Limit points of $\omega_{1}$ are those elements which are not of the form $i+1$. The least element of $\omega_{1}$ is called 0 .

We will use a straighforward bookkeeping argument to take care of $5.4(2-4)$.

By CH, let $\left(f_{i}: i \in \omega_{1}\right)$ enumerate all growth functions. Let $H: \omega_{1} \times \omega_{1} \rightarrow \omega_{1}$ be a bijection satisfying $H(\alpha, \beta) \geq \alpha$ for all $\alpha, \beta \in \omega_{1}$, and let $\left(H_{1}, H_{2}\right)$ be the inverse functions, i.e., $H\left(H_{1}(\gamma), H_{2}(\gamma)\right)=\gamma$ for all $\gamma \in \omega_{1}$.

Now define a $\leqq^{*}$-decreasing sequence $\left(s_{i}: i \in \omega_{1}\right)$ as follows:

- $s_{0}$ is arbitrary.

- Assume that $\left(s_{j}: j \leq i\right)$ is already defined. We will define $s_{i+1}$. Let $\left\{c_{j}^{i}: j \in \omega_{1}\right\}$ be the set of all coloring functions of $s_{i}$. (Again, such an enumeration exists because we are assuming $\mathrm{CH}$.)

Now let $s_{i+1} \leqq s_{i}$ be such that

(a) $s_{i+1}$ is $f_{i}$-strong,

(b) $\left(\operatorname{root}\left(s_{i}\right) \cap s_{i+1}\right)+1 \preccurlyeq s_{i+1} \operatorname{root}\left(s_{i+1}\right)$,

(c) $s_{i+1}$ is $c_{H_{2}(i)}^{H_{1}(i)}$-homogeneous. (Recall that $H_{1}(i) \leq i$, so $s_{H_{1}(i)}$ is already defined; $c_{H_{2}(i)}^{H_{1}(i)}$ is a coloring of $s_{H_{1}(i)}$.)

$s_{i+1}$ can easily be obtained in 3 steps $s_{i} \geqq s_{i}^{(\mathrm{a})} \geqq s_{i}^{(\mathrm{b})} \geqq s_{i}^{(\mathrm{c})}=s_{i+1}$, where in each step we satisfy one of the demands (a), (b), (c). For example, (b) can be realized by using the "gluing" step from 3.7

- If $j$ is a limit point, then let $s_{j}$ be any zoo satisfying $\forall i<j: s_{j} \leqq * s_{i}$. This is possible by 3.15, (See also Remark 6.1.) 
The following facts are easy and well known:

\subsection{Fact.}

(1) Assume CH. Then there is a unique linear order $\left(D_{1}, \leq\right)$ with the following properties:

- $D_{1}$ has a smallest element but no largest element.

- $D_{1}$ is of cardinality $\aleph_{1}$.

- $D_{1}$ is $\aleph_{1}$-dense, i.e., between any two elements there are uncountably many elements.

- Every countable subset of $D_{1}$ is bounded, and moreover:

For any two countable sets $C, C^{\prime} \subseteq D_{1}$ with $C \leq C^{\prime}$ [i.e., $\forall x \in C \forall y \in$ $\left.C^{\prime}: x \leq y\right]$ there is $c$ with $C \leq\{c\} \leq C^{\prime}$.

(2) Assuming $\mathrm{CH}$, there is also a unique linear order $D_{2}$ with the following properties:

- $D_{2}$ has a smallest element but no largest element.

- Every element of $D_{2}$ has a direct successor.

- Every element of $D_{2}$ (except for the minimal element) has a direct predecessor.

- Factoring $D_{2}$ by the relation

$$
x \sim y \quad \Longleftrightarrow \quad \text { the interval }[x, y] \text { is finite }
$$

yields $D_{1}$.

Proof. A back-and-forth argument, similar to Cantor's theorem characterizing the rationals as the unique dense linear order without endpoints.

$D_{2}$ can be obtained as the lexicographic order on

$$
\left(\left\{\min D_{1}\right\} \times \mathbb{N}\right) \cup\left(D_{1} \backslash\left\{\min D_{1}\right\}\right) \times \mathbb{Z} .
$$

5.8. Conclusion. Assume $\mathrm{CH}$.

Let $\vec{s}=\left(s_{i}: i \in \omega_{1}\right)$ be sufficiently generic, and let $U$ be the filter generated by $\left(\operatorname{ext}\left(s_{i}\right): i \in \omega_{1}\right)$. Then

(a) $U$ is an ultrafilter.

(b) $(\mathcal{F} \vec{s}, \preccurlyeq \vec{s})$ is order isomorphic to $D_{2}$.

(c) The set $\mathcal{G} / \sim_{U}$, ordered by $\leq_{U}$, is order isomorphic to $D_{2}$.

(d) Letting $\langle U\rangle$ be the clone generated by $\mathcal{C}_{\text {id }} \cup\left\{h_{A}: A \in U\right\}$, the interval $[\langle U\rangle, \mathcal{O}]$ in the clone lattice is order isomorphic to the Dedekind completion of $D_{2}$.

Proof. (a) is not needed and left to the reader.

(b) By 5.5 and 5.7

(c) We define a map $K$ from $\mathcal{G}$ to the set of *fronts in $\vec{s}$ :

For any $f \in \mathcal{G}$, pick $i \in I$ such that $s_{i}$ is almost $f$-strong and gauges $f$. Let

$$
K(f)=F\left(s_{i}, f\right) .
$$

(Recall that for all $j \geq i, F\left(s_{j}, f\right) \approx_{s_{j}}^{*} K(f) \cap s_{j}$.)

We now claim that

(*) $f \leq_{U} g$ iff $K(f) \preccurlyeq_{\vec{s}}^{*} K(g)$. 
To prove this claim, fix $f$ and $g$. Pick some sufficiently large $j \in \omega_{1}$. By 5.5 one of the following cases holds:

(i) $K(f) \approx_{\vec{s}}^{*} K(g)$, so $F\left(s_{j}, f\right) \approx_{s_{j}}^{*} F\left(s_{j}, g\right)$,

(ii) $K(f)+1 \preccurlyeq_{\vec{s}}^{*} K(g)$, so $F\left(s_{j}, f\right) \prec_{s_{j}}^{*} F\left(s_{j}, g\right)$,

(iii) $K(g)+1 \preccurlyeq_{\vec{s}}^{*} K(f)$, so $F\left(s_{j}, g\right) \prec_{s_{j}}^{*} F\left(s_{j}, f\right)$.

In the first case, 4.15 implies $f \leq_{\operatorname{ext}\left(s_{j}\right)} g$, hence $f \leq_{U} g$ and also $g \leq_{U} f$, so $f \sim_{U} g$.

In the second case, we have $F\left(s_{i}, f\right) \prec_{s_{i}}^{*} F\left(s_{i}, g\right)$ for all $i \geq j$, so by 4.16

$$
\forall i \geq j: \quad f \leq_{\operatorname{ext}\left(s_{i}\right)} g \text { and } g \not_{\mathbf{e x t}\left(s_{i}\right)} f
$$

which implies $f \leq_{U} g$ but $g \not_{U} f$.

In the third case we get similarly $g \leq_{U} f$ and $f \mathbb{L}_{U} g$.

So in each case the desired equivalence holds, and $(*)$ is proved.

Hence $K$ induces an order isomorphism between $\mathcal{G} / U$ and $\mathcal{F} \vec{s}$.

(d) follows from our discussion in Section 1 .

\section{Discussion}

6.1. Remark. It is clear that the full strength of $\mathrm{CH}$ is not necessary for this construction. Martin's axiom MA (even the version for $\sigma$-centered forcing notions) is easily shown to imply an analogue of Corollary 3.15 in which "countable" is replaced by "of size $<2^{\aleph_{0}}$ ". This allows us to modify the construction in 5.6 to a transfinite induction of length $2^{\aleph_{0}}$, which shows that already MA implies the existence of a sufficiently generic sequence.

Thus, the conclusion of our theorem is also consistent with the negation of $\mathrm{CH}$.

To analyse our proof, the following cardinal may be helpful.

6.2. Definition. In analogy to the usual "tower" number $\mathfrak{t}$ (see [17, 18]) we let $\mathfrak{t}_{z}$ the smallest cardinal for which a counterexample to corollary 3.15 exists, i.e., the smallest cardinality of a linearly quasiordered set $Z$ of zoos such that there is no zoo $t$ with $\forall s \in Z: t \leq^{*} s$.

A cardinal $\mathfrak{p}_{z}$ can be defined similarly.

What we really showed was:

(1) $\mathfrak{t}_{z} \geq \aleph_{1}$ (3.15).

(2) If $\mathfrak{t}_{z}=2^{\aleph_{0}}$, then

(*) there is an ultrafilter $U$ such that $\mathcal{G} / U$ is linearly ordered without last element.

(3) If $(*)$, then

$(* *) C l(\mathbb{N})$ is not dually atomic.

This leads naturally to the following questions:

\subsection{Problems.}

(1) Is $\mathfrak{t}_{z}=2^{\aleph_{0}}$ provable in ZFC?

(2) Is $(*)$ (or a sufficiently strong variant) provable in ZFC?

(3) Is $(* *)$ provable in ZFC?

Note that "yes" to questions 6.3(1) or 6.3 (2) also implies "yes" to the subsequent question(s); however, while it will be interesting to compare $\mathfrak{t}_{z}$ and $\mathfrak{p}_{z}$ to the other 
well-known cardinal characteristics of the continuum ([17, 18]) we suspect that $\mathfrak{t}_{z}<2^{\aleph_{0}}$ is consistent with ZFC, which makes such a discussion useless as far as problem 6.3 $[3)$ is concerned.

Note also that our whole construction took part within the interval $\left[\mathcal{C}_{\mathrm{id}}, \mathcal{O}\right]$ of the clone lattice. Even the strongest possible negative answer to6.3(2), "Consistently, $\left[\mathcal{C}_{\mathrm{id}}, \mathcal{O}\right]$ is dually atomic", would not exclude the possibility that dual atoms may be absent from other intervals $[\mathcal{C}, \mathcal{O}]$.

\section{ACKNOWLEDGMENT}

We are grateful to Lutz Heindorf for his suggestions that led to an improvement over an earlier version of the paper, to the referee for his/her insightful comments, and to Günther Eigenthaler for his meticulous proofreading.

\section{REFERENCES}

[1] V. A. Buevich. A new version of the proof of completeness criterion for $k$-valued logic functions. Discrete Math. Appl., 6(5):505-530, 1996. MR98g:03067

[2] G. P. Gavrilov. Certain conditions for completeness in countable-valued logic. Dokl. Akad. Nauk SSSR, 128:21-24, 1959. in Russian. MR21:6327

[3] G.P. Gavrilov. Ueber funktionale Vollstaendigkeit in der abzaehlbar-wertigen Logik. Probl. Kibernetiki, 15:5-64, 1965. MR37:5085

[4] Martin Goldstern and Saharon Shelah. Clones on regular cardinals. Fundamenta Mathematicae, 173:1-20, 2002. math.RA/0005273. MR2003c:08006

[5] Martin Goldstern and Saharon Shelah. Large Intervals in the Clone Lattice. Algebra Universalis, 200x. math.RA/0208066.

[6] Lutz Heindorf. The maximal clones on countable sets that include all permutations. Algebra universalis, 48:209-222, 2002. MR2003g:08005

[7] H. Machida and I. G. Rosenberg. A "large" essentially minimal clone over an infinite set. In Proceedings of the International Conference on Algebra, Part 3 (Novosibirsk, 1989), volume 131 of Contemp. Math., pages 159-167, Providence, RI, 1992. Amer. Math. Soc. MR93f:08005

[8] R. Pöschel and L. A. Kalužnin. Funktionen- und Relationenalgebren, volume 15 of Mathematische Monographien [Mathematical Monographs]. VEB Deutscher Verlag der Wissenschaften, Berlin, 1979. Ein Kapitel der diskreten Mathematik. [A chapter in discrete mathematics]. MR:81f:03075

[9] R. W. Quackenbush. A new proof of Rosenberg's primal algebra characterization theorem. Colloquia Mathematica Societatis János Bolyai, 28:603-634, 1971. MR83f:08012

[10] I. G. Rosenberg. Über die funktionale Vollständigkeit in den mehrwertigen Logiken. Rozpravy Československé Akad. věd, Ser. Math. Nat. Sci., 80:3-93, 1970. MR 45:1732

[11] I. G. Rosenberg. Some maximal closed classes of operations on infinite sets. Math. Ann., 212:157-164, 1974/75. MR50:4452

[12] I. G. Rosenberg. The set of maximal closed classes of operations on an infinite set $A$ has cardinality $2^{2^{|A|}}$. Arch. Math. (Basel), 27(6):561-568, 1976. MR $55: 2711$

[13] Ivo G. Rosenberg and Dietmar Schweigert. Locally maximal clones. Elektron. Informationsverarb. Kybernet., 18(7-8):389-401, 1982. MR85b:08006

[14] Andrzej Roslanowski and Saharon Shelah. Norms on possibilities I: forcing with trees and creatures. Memoirs of the American Mathematical Society, 141(671), 1999. math.LO/9807172. MR2000c:03036

[15] Saharon Shelah. On cardinal invariants of the continuum. In Axiomatic set theory (Boulder, Colo., 1983), volume 31 of Contemp. Mathematics, pages 183-207. Amer. Math. Soc., Providence, RI, 1984. Proceedings of the Conference in Set Theory, Boulder, June 1983; eds. J. Baumgartner, D. Martin, and S. Shelah. MR 86b:03064

[16] Ágnes Szendrei. Clones in universal algebra. Presses de l'Université de Montréal, Montreal, Que., 1986. MR87m:08005 
[17] Eric K. van Douwen. The integers and topology. In K. Kunen and J. E. Vaughan, editors, Handbook of Set-Theoretic Topology, pages 111-167. Elsevier Science Publishers, 1984. MR $87 \mathrm{f}: 54008$

[18] Jerry E. Vaughan. Small uncountable cardinals and topology, pages 195-218. North-Holland, Amsterdam, 1990. With an appendix by S. Shelah. MR92c:54001

Institute of Discrete Mathematics and Geometry, Vienna University of Technology, A-1040 Vienna, Austria

E-mail address: goldstern@tuwien.ac.at

$U R L:$ http://www.tuwien.ac.at/goldstern/

Institute of Mathematics, Hebrew University of Jerusalem, 91904 Jerusalem, Israel - And - Department of Mathematics, Rutgers University, New Brunswick, New Jersey 08854

E-mail address: shelah@math.huji.ac.il

$U R L:$ http://shelah.logic at 